\title{
Facile Access to Pentavalent Uranium Organometallics: One Electron Oxidation of Uranium(IV) Imido Complexes with Copper(I) Salts
}

\author{
Christopher R. Graves, Brian L. Scott, David E. Morris*, and Jaqueline L. Kiplinger* \\ Los Alamos National Laboratory, Los Alamos, NM 87545, USA \\ Fax: +01 505667 9905; Tel: +01 505665 9553; E-mail: kiplinger@lanl.gov
}

\section{Electronic Supplementary Information:}

- General Synthetic Considerations $\quad$ S2

- Synthesis of $\left(\mathrm{C}_{5} \mathrm{Me}_{5}\right)_{2} \mathrm{U}\left(=\mathrm{N}-2,4,6-{ }^{t} \mathrm{Bu}_{3}-\mathrm{C}_{6} \mathrm{H}_{2}\right)(\mathrm{I})(3) \quad$ S3

- Synthesis of $\left(\mathrm{C}_{5} \mathrm{Me}_{5}\right)_{2} \mathrm{U}\left(=\mathrm{N}-2,6-{ }^{i} \mathrm{Pr}_{2}-\mathrm{C}_{6} \mathrm{H}_{3}\right)(\mathrm{I})(\mathbf{4}) \quad$ S3

- Synthesis of $\left(\mathrm{C}_{5} \mathrm{Me}_{5}\right)_{2} \mathrm{U}\left(=\mathrm{N}-2,6-{ }^{i} \mathrm{Pr}_{2}-\mathrm{C}_{6} \mathrm{H}_{3}\right)(\mathrm{OTf})(\mathbf{5}) \quad$ S3-S4

- Synthesis of $\left(\mathrm{C}_{5} \mathrm{Me}_{5}\right)_{2} \mathrm{U}\left(=\mathrm{N}-2,6-{ }^{i} \mathrm{Pr}_{2}-\mathrm{C}_{6} \mathrm{H}_{3}\right)(\mathrm{SPh})(6) \quad$ S4

- Figure S1. Molecular Structure of Complex $3 \quad$ S5

- Table S1. Crystal Data and Structure Refinement for $\left(\mathrm{C}_{5} \mathrm{Me}_{5}\right)_{2} \mathrm{U}\left(=\mathrm{N}-2,4,6-{ }^{\mathrm{t}} \mathrm{Bu}_{3}-\mathrm{C}_{6} \mathrm{H}_{2}\right)(\mathrm{I})(3) \quad$ S6

- Table S2. Bond Lengths $[\AA]$ and Angles $\left[{ }^{\circ}\right]$ for $\left(\mathrm{C}_{5} \mathrm{Me}_{5}\right)_{2} \mathrm{U}\left(=\mathrm{N}-2,4,6-{ }^{t} \mathrm{Bu}_{3}-\mathrm{C}_{6} \mathrm{H}_{2}\right)(\mathrm{I})(3) \quad$ S7-S9

- Figure S2 Molecular Structure of Complex $4 \quad$ S10

- Table S3. Crystal Data and Structure Refinement for $\left(\mathrm{C}_{5} \mathrm{Me}_{5}\right)_{2} \mathrm{U}\left(=\mathrm{N}-2,6-{ }^{i} \mathrm{Pr}_{2}-\mathrm{C}_{6} \mathrm{H}_{3}\right)(\mathrm{I})$ (4) $\quad$ S11

- Table S4. Bond Lengths $[\AA]$ and Angles $\left[{ }^{\circ}\right]$ for $\left(\mathrm{C}_{5} \mathrm{Me}_{5}\right)_{2} \mathrm{U}\left(=\mathrm{N}-2,6-{ }^{i} \mathrm{Pr}_{2}-\mathrm{C}_{6} \mathrm{H}_{3}\right)(\mathrm{I})(4) \quad$ S12-S17

$\begin{array}{lr}\text { - Figure S3. Molecular Structure of Complex } 5 & \text { S18 }\end{array}$

- Table S5. Crystal Data and Structure Refinement for $\left(\mathrm{C}_{5} \mathrm{Me}_{5}\right)_{2} \mathrm{U}\left(=\mathrm{N}-2,6-{ }^{i} \mathrm{Pr}_{2}-\mathrm{C}_{6} \mathrm{H}_{3}\right)\left(\mathrm{OSO}_{2} \mathrm{CF}_{3}\right)(\mathbf{5}) \quad$ S19

- Table S6. Bond Lengths $[\AA]$ and Angles $\left[{ }^{\circ}\right]$ for $\left(\mathrm{C}_{5} \mathrm{Me}_{5}\right)_{2} \mathrm{U}\left(=\mathrm{N}-2,6-{ }^{i} \mathrm{Pr}_{2}-\mathrm{C}_{6} \mathrm{H}_{3}\right)\left(\mathrm{OSO}_{2} \mathrm{CF}_{3}\right)(\mathbf{5}) \quad$ S20-S22

- Figure S4 Molecular Structure of Complex $6 \quad$ S23

- Table S7. Crystal Data and Structure Refinement for $\left(\mathrm{C}_{5} \mathrm{Me}_{5}\right)_{2} \mathrm{U}\left(=\mathrm{N}-2,6-{ }^{i} \mathrm{Pr}_{2}-\mathrm{C}_{6} \mathrm{H}_{3}\right)(\mathrm{SPh})(6) \quad$ S24

- Table S8. Bond Lengths $[\AA]$ and Angles $\left[{ }^{\circ}\right]$ for $\left(\mathrm{C}_{5} \mathrm{Me}_{5}\right)_{2} \mathrm{U}\left(=\mathrm{N}-2,6-{ }^{i} \mathrm{Pr}_{2}-\mathrm{C}_{6} \mathrm{H}_{3}\right)(\mathrm{SPh})(6) \quad$ S25-S27 
General Synthetic Considerations: Reactions and manipulations were performed at $20{ }^{\circ} \mathrm{C}$ in a recirculating Vacuum Atmospheres NEXUS model inert atmosphere $\left(\mathrm{N}_{2}\right)$ drybox equipped with a 40CFM Dual Purifier NI-Train, or using standard Schlenk and high vacuum line techniques. Glassware was dried overnight at $150{ }^{\circ} \mathrm{C}$ before use. All NMR spectra were obtained in $\mathrm{C}_{6} \mathrm{D}_{6}$ using a Bruker Avance $300 \mathrm{MHz}$ spectrometer at ambient temperature. Chemical shifts for ${ }^{1} \mathrm{H}$ NMR spectra were referenced to solvent impurities and ${ }^{19} \mathrm{~F}$ NMR resonances were referenced to $\mathrm{CFCl}_{3}$ at $\delta 0.00 \mathrm{ppm}$. Melting points were determined with a Met-Temp II capillary melting point apparatus equipped with a Fluke $50 \mathrm{~S} \mathrm{~K} / \mathrm{J}$ thermocouple using capillary tubes flame-sealed under nitrogen; values are uncorrected. Mass spectrometric (MS) analyses were obtained at the University of California, Berkeley Mass Spectrometry Facility, using a VG ProSpec (EI) mass spectrometer. Elemental analyses were performed at the University of California, Berkeley Microanalytical Facility, on a Perkin-Elmer Series II 2400 CHNS analyzer. Xray data were collected using a Bruker APEX2 diffractometer. Structural solution and refinement was achieved using the SHELXL97 program suite. Details regarding data collection are provided in the CIF files.

Benzene- $d_{6}$ was obtained from Cambridge Isotope Laboratories and was purified by passage through activated alumina and storage over activated $4 \AA$ molecular sieves prior to use. Celite (Aldrich) and alumina (Brockman I, Aldrich) were dried under reduced pressure at $250{ }^{\circ} \mathrm{C}$ for $48 \mathrm{~h}$ prior to use. Anhydrous toluene (Aldrich) was dried over KH for $24 \mathrm{~h}$, passed through a column of activated alumina, and stored over activated $4 \AA$ molecular sieves prior to use. $\left(\mathrm{C}_{5} \mathrm{Me}_{5}\right)_{2} \mathrm{U}\left(=\mathrm{N}-2,4,6{ }^{\mathrm{t}}{ }^{-} \mathrm{Bu}_{3}-\mathrm{C}_{6} \mathrm{H}_{2}\right)(\mathbf{1})^{1}$ and $\left(\mathrm{C}_{5} \mathrm{Me}_{5}\right)_{2} \mathrm{U}\left(=\mathrm{N}-2,6-{ }^{i} \mathrm{Pr}_{2}-\mathrm{C}_{6} \mathrm{H}_{3}\right)(\mathrm{THF})(\mathbf{2})^{2}$ were prepared according to literature procedures.

Caution: Depleted uranium (primary isotope ${ }^{238} \mathrm{U}$ ) is a weak $\alpha$-emitter $(4.197 \mathrm{MeV}$ ) with a half-life of 4.47 x $10^{9}$ years; manipulations and reactions should be carried out in monitored fume hoods or in an inert atmosphere drybox in a radiation laboratory equipped with $\alpha$ - and $\beta$-counting equipment.

References: (1) Arney, D. S. J.; Burns, C. J. J. Am Chem. Soc. 1995, 117, 9448-9460. (2) Arney, D. S. J.; Burns, C. J. J. Am Chem. Soc. 1993, 115, 9840-9841. 
Synthesis of $\left(\mathbf{C}_{5} \mathbf{M e}_{5}\right)_{2} \mathbf{U}\left(=\mathbf{N}-\mathbf{2 , 4 , 6}{ }^{t}{ }^{t} \mathbf{B u}_{3}-\mathbf{C}_{6} \mathbf{H}_{2}\right)(\mathbf{I})$ (3): To a stirring solution of $\mathbf{1}$ (1.0 g, $\left.1.3 \mathrm{mmol}\right)$ in toluene (50 $\mathrm{mL})$ was added $\mathrm{CuI}(1.2 \mathrm{~g}, 6.5 \mathrm{mmol})$ as an off-white power. The reaction vessel was sealed and the solution was stirred at room temperature. After $12 \mathrm{~h}$, the reaction was filtered through a Celite-packed coarse-porosity frit and the volatiles were removed under reduced pressure. The resulting dark brown solid was taken up into hexanes and re-filtered through a Celite-packed coarse-porosity frit. Solvents were removed from the filtrate to give $\mathbf{3}$ as a dark brown solid. Yield: $0.89 \mathrm{~g}, 1.0 \mathrm{mmol}(78 \%)$. Analytically pure samples were obtained by recrystallization from hexanes at $-30{ }^{\circ} \mathrm{C} .{ }^{1} \mathrm{H}$ NMR $\left(\mathrm{C}_{6} \mathrm{D}_{6}, 300 \mathrm{MHz}, 298 \mathrm{~K}\right): \delta 30.21(1 \mathrm{H}, \mathrm{Ar}-\mathrm{H}), 25.81(1 \mathrm{H}, \mathrm{Ar}-\mathrm{H}), 17.32\left(9 \mathrm{H}, \mathrm{C}\left(\mathrm{CH}_{3}\right)_{3}\right)$, 6.42-5.54 (39H, $\left.\left(\mathrm{C}_{5}\left(\mathrm{CH}_{3}\right)_{5}\right)_{2}+\mathrm{C}\left(\mathrm{CH}_{3}\right)_{3}\right),-1.97\left(9 \mathrm{H}, \mathrm{C}\left(\mathrm{CH}_{3}\right)_{3}\right)$. Anal. Calcd for $\mathrm{C}_{38} \mathrm{H}_{59} \mathrm{NIU}$ (mol. wt. 894.82): $\mathrm{C}$, 51.01; H, 6.65; N, 1.57. Found: C, 50.69; H, 6.53; N, 1.67. Mp $=194{ }^{\circ} \mathrm{C} . \mathrm{MS}(\mathrm{EI}, 70 \mathrm{eV}): \mathrm{m} / \mathrm{z} 896\left(\mathrm{M}^{+}\right) . \mathrm{U}(\mathrm{VI} / \mathrm{V})$ $\mathrm{E}_{1 / 2}=0.06 \mathrm{~V}, \mathrm{U}(\mathrm{V} / \mathrm{IV}) \mathrm{E}_{1 / 2}=-1.31 \mathrm{~V}\left(\mathrm{vs} .\left[\left(\mathrm{C}_{5} \mathrm{H}_{5}\right)_{2} \mathrm{Fe}\right]^{+/ 0}\right.$ in THF/0.1 M $\left.\left[\mathrm{Bu}_{4} \mathrm{~N}\right]\left[\mathrm{B}\left(\mathrm{C}_{6} \mathrm{~F}_{5}\right)_{4}\right]\right)$.

Synthesis of $\left(\mathrm{C}_{5} \mathrm{Me}_{5}\right)_{2} \mathrm{U}\left(=\mathbf{N}-\mathbf{2}, 6-{ }^{i} \mathrm{Pr}_{2}-\mathrm{C}_{6} \mathrm{H}_{3}\right)(\mathrm{I})$ (4): An analogous procedure was followed as in the synthesis of 3 using $2(1.0 \mathrm{~g}, 1.3 \mathrm{mmol})$ as the starting material. Product 4 was obtained in $81 \%$ yield $(0.89 \mathrm{~g}, 1.1 \mathrm{mmol})$ as a dark brown solid. Analytically pure samples were obtained by recrystallization from hexanes at $-30{ }^{\circ} \mathrm{C} .{ }^{1} \mathrm{H}-\mathrm{NMR}\left(\mathrm{C}_{6} \mathrm{D}_{6}\right.$, $300 \mathrm{MHz}, 298 \mathrm{~K}): \delta 57.95$ (b, 1H, $\left.\mathrm{CH}\left(\mathrm{CH}_{3}\right)_{2}\right), 30.86(1 \mathrm{H}, \mathrm{Ar}-\mathrm{H}), 23.95(1 \mathrm{H}, \mathrm{Ar}-\mathrm{H}), 13.81\left(6 \mathrm{H}, \mathrm{CH}\left(\mathrm{CH}_{3}\right)_{2}\right), 5.78$ (30H, $\left.\left(\mathrm{C}_{5}\left(\mathrm{CH}_{3}\right)_{5}\right)_{2}\right),-5.14\left(6 \mathrm{H}, \mathrm{CH}\left(\mathrm{CH}_{3}\right)_{2}\right),-7.26(1 \mathrm{H}, \mathrm{Ar}-\mathrm{H}),-9.25\left(\mathrm{~b}, 1 \mathrm{H}, \mathrm{CH}\left(\mathrm{CH}_{3}\right)_{2}\right)$. Anal. Calcd for $\mathrm{C}_{32} \mathrm{H}_{47} \mathrm{NIU}$ (mol. wt. 810.66): C, 47.41; H, 5.84; N, 1.73. Found: C, 47.23; H, 5.78; N, 1.75. Mp $=261{ }^{\circ} \mathrm{C} . \quad \mathrm{MS}$ (EI, $70 \mathrm{eV}$ ): $\mathrm{m} / \mathrm{z} 810\left(\mathrm{M}^{+}\right) . \mathrm{U}(\mathrm{VI} / \mathrm{V}) \mathrm{E}_{1 / 2}=0.11 \mathrm{~V}, \mathrm{U}(\mathrm{V} / \mathrm{IV}) \mathrm{E}_{1 / 2}=-1.37 \mathrm{~V}\left(\mathrm{vs} .\left[\left(\mathrm{C}_{5} \mathrm{H}_{5}\right)_{2} \mathrm{Fe}\right]^{+/ 0}\right.$ in THF/0.1 M $\left.\left[{ }^{\mathrm{n}} \mathrm{Bu} \mathrm{u}_{4} \mathrm{~N}\right]\left[\mathrm{B}\left(\mathrm{C}_{6} \mathrm{~F}_{5}\right)_{4}\right]\right)$.

Synthesis of $\left(\mathbf{C}_{5} \mathbf{M e}_{5}\right)_{2} \mathbf{U}\left(=\mathbf{N}-\mathbf{2}, 6-{ }^{i} \mathbf{P r}_{2}-\mathbf{C}_{6} \mathbf{H}_{3}\right)(\mathbf{O T f})$ (5): To a stirring solution of $\mathbf{2}(0.25 \mathrm{~g}, 0.33 \mathrm{mmol})$ in toluene $(250 \mathrm{~mL})$ was added $\left(\mathrm{CuOSO}_{2} \mathrm{CF}_{3}\right)_{2}$ toluene $(0.17 \mathrm{~g}, 0.33 \mathrm{mmol})$ as an off-white power. The reaction vessel was sealed and the reaction was stirred at room temperature. After $1 \mathrm{~h}$, the reaction was filtered through a Celite-packed coarse-porosity frit and the volatiles were removed under reduced pressure. The resulting dark brown solid was taken up into hexanes and re-filtered through a Celite-packed coarse-porosity frit. Solvents were removed from the filtrate to give 5 as a dark brown solid. Yield: $0.21 \mathrm{~g}, 0.25 \mathrm{mmol}, 76 \%$. Analytically pure samples were obtained by either recrystallization from hexanes or a toluene/pentane mixture at $-30{ }^{\circ} \mathrm{C} .{ }^{1} \mathrm{H}-\mathrm{NMR}\left(\mathrm{C}_{6} \mathrm{D}_{6}, 300 \mathrm{MHz}, 298 \mathrm{~K}\right): \delta$ $60.43\left(\mathrm{~b}, 1 \mathrm{H}, \mathrm{CH}\left(\mathrm{CH}_{3}\right)_{2}\right), 30.49(1 \mathrm{H}, \mathrm{Ar}-\mathrm{H}), 22.86(1 \mathrm{H}, \mathrm{Ar}-\mathrm{H}), 14.36\left(6 \mathrm{H}, \mathrm{CH}\left(\mathrm{CH}_{3}\right)_{2}\right), 5.98\left(30 \mathrm{H},\left(\mathrm{C}_{5}\left(\mathrm{CH}_{3}\right)_{5}\right)_{2}\right)$, $6.42\left(6 \mathrm{H}, \mathrm{CH}\left(\mathrm{CH}_{3}\right)_{2}\right),-11.58(1 \mathrm{H}, \mathrm{Ar}-\mathrm{H}),-18.49\left(\mathrm{~b}, 1 \mathrm{H}, \mathrm{CH}\left(\mathrm{CH}_{3}\right)_{2}\right) .{ }^{19} \mathrm{~F}$ NMR: $\delta-70.64$. Anal. Calcd for $\mathrm{C}_{33} \mathrm{H}_{47} \mathrm{NO}_{3} \mathrm{~F}_{3} \mathrm{SU}$ (mol. wt. 832.82): C, 47.59; H, 5.69; N, 1.68. Found: C, 47.26; H, 5.99; N, 1.93. $\mathrm{Mp}=22{ }^{\circ} \mathrm{C}$. MS (EI, $70 \mathrm{eV}): \mathrm{m} / \mathrm{z} 832\left(\mathrm{M}^{+}\right) . \mathrm{U}(\mathrm{VI} / \mathrm{V}) \mathrm{E}_{1 / 2}=0.37 \mathrm{~V}, \mathrm{U}(\mathrm{V} / \mathrm{IV}) \mathrm{E}_{1 / 2}=-1.43 \mathrm{~V}\left(\mathrm{vs} .\left[\left(\mathrm{C}_{5} \mathrm{H}_{5}\right)_{2} \mathrm{Fe}\right]^{+/ 0}\right.$ in THF/0.1 M 
$\left.\left[{ }^{\mathrm{n}} \mathrm{Bu} \mathrm{u}_{4} \mathrm{~N}\right]\left[\mathrm{B}\left(\mathrm{C}_{6} \mathrm{~F}_{5}\right)_{4}\right]\right)$.

Synthesis of $\left(\mathbf{C}_{5} \mathbf{M e}_{5}\right)_{2} \mathbf{U}\left(=\mathbf{N}-2,6-{ }^{i} \mathbf{P r}_{2}-\mathbf{C}_{6} \mathbf{H}_{3}\right)(\mathbf{S P h})$ (6): To a stirring solution of $\mathbf{2}(0.25 \mathrm{~g}, 0.33 \mathrm{mmol})$ in toluene $(100 \mathrm{~mL})$ was added $\mathrm{CuSPh}(0.29 \mathrm{~g}, 1.65 \mathrm{mmol})$ as an pale yellow power. The reaction vessel was sealed and the reaction was stirred at $75{ }^{\circ} \mathrm{C}$. After $12 \mathrm{~h}$, the reaction was filtered through a Celite-packed coarse-porosity frit and the volatiles were removed under reduced pressure. The resulting dark brown solid was taken up into hexanes and re-filtered through a Celite-packed coarse-porosity frit. Solvents were removed from the filtrate to give $\mathbf{6}$ as a dark brown solid. Yield: $0.24 \mathrm{~g}, 0.30 \mathrm{mmol}, 90 \%$. Analytically pure samples were obtained by recrystallization from hexanes at $-30{ }^{\circ} \mathrm{C} . \quad{ }^{1} \mathrm{H}-\mathrm{NMR}\left(\mathrm{C}_{6} \mathrm{D}_{6}, 300 \mathrm{MHz}, 298 \mathrm{~K}\right): \delta 51.33\left(\mathrm{~b}, 1 \mathrm{H}, \mathrm{CH}\left(\mathrm{CH}_{3}\right)_{2}\right), 29.45(1 \mathrm{H}, \mathrm{Ar}-\mathrm{H}), 24.18(1 \mathrm{H}$, $\mathrm{Ar}-\mathrm{H}), 11.92\left(6 \mathrm{H}, \mathrm{CH}\left(\mathrm{CH}_{3}\right)_{2}\right), 6.31(2 \mathrm{H}, \mathrm{Ar}-\mathrm{H}) ; 4,57\left(30 \mathrm{H},\left(\mathrm{C}_{5}\left(\mathrm{CH}_{3}\right)_{5}\right)_{2}\right), 1.42(1 \mathrm{H}, \mathrm{Ar}-\mathrm{H}), 1.24(1 \mathrm{H}, \mathrm{Ar}-\mathrm{H}), 0.89$ $(1 \mathrm{H}, \mathrm{Ar}-\mathrm{H}),-2.47\left(6 \mathrm{H}, \mathrm{CH}\left(\mathrm{CH}_{3}\right)_{2}\right),-4.74(1 \mathrm{H}, \mathrm{Ar}-\mathrm{H}),-7.95\left(\mathrm{~b}, 1 \mathrm{H}, \mathrm{CH}\left(\mathrm{CH}_{3}\right)_{2}\right)$. Anal. Calcd for $\mathrm{C}_{38} \mathrm{H}_{52} \mathrm{NSU}(\mathrm{mol}$. wt. 792.92): C, 57.56; H, 6.61; N, 1.77. Found: C, 57.40; H, 6.58; N, 1.72. Mp = $181{ }^{\circ} \mathrm{C} . \mathrm{MS}(\mathrm{EI}, 70 \mathrm{eV}): \mathrm{m} / \mathrm{z} 792$ $\left(\mathrm{M}^{+}\right) . \mathrm{U}(\mathrm{VI} / \mathrm{V}) \mathrm{E}_{1 / 2}=0.00 \mathrm{~V}, \mathrm{U}(\mathrm{V} / \mathrm{IV}) \mathrm{E}_{1 / 2}=-1.45 \mathrm{~V}\left(\mathrm{vs} .\left[\left(\mathrm{C}_{5} \mathrm{H}_{5}\right)_{2} \mathrm{Fe}\right]^{+/ 0}\right.$ in THF/0.1 M $\left.\left[{ }^{\mathrm{n}} \mathrm{Bu}_{4} \mathrm{~N}\right]\left[\mathrm{B}\left(\mathrm{C}_{6} \mathrm{~F}_{5}\right)_{4}\right]\right)$. 
Figure S1. Molecular structure of complex $\mathbf{3}$ with thermal ellipsoids at the $50 \%$ probability level.

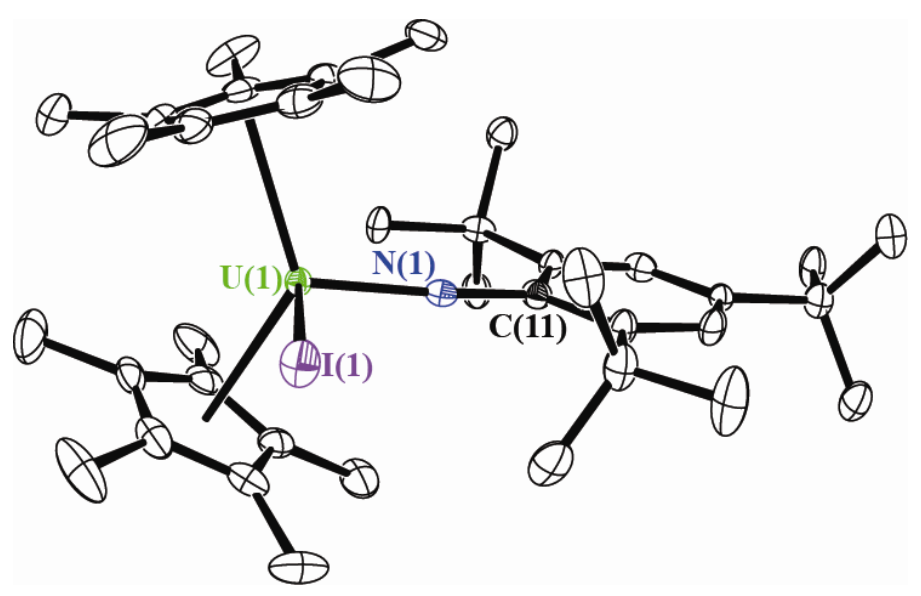


Table S1. Crystal Data and Structure Refinement for $\left(\mathrm{C}_{5} \mathrm{Me}_{5}\right)_{2} \mathrm{U}\left(=\mathrm{N}-2,4,6-{ }^{t} \mathrm{Bu}_{3}-\mathrm{C}_{6} \mathrm{H}_{2}\right)(\mathrm{I})(3)$.

Empirical formula

Formula weight

Temperature

Wavelength

Crystal system

Space group

Unit cell dimensions

Volume

Z

Density (calculated)

Absorption coefficient

$\mathrm{F}(000)$

Crystal size

$\theta$ Range for data collection

Index ranges

Reflections collected

Independent reflections

Completeness to $\theta=25.00^{\circ}$

Max. and min. transmission

Refinement method

Data / restraints / parameters

Goodness-of-fit on $\mathrm{F}^{2}$

Final $R$ indices $[\mathrm{I}>2 \sigma(\mathrm{I})]$

$\mathrm{R}$ indices (all data)

Largest diff. peak and hole
$\mathrm{C}_{41} \mathrm{H}_{73} \mathrm{INU}\left(\mathrm{C}_{35} \mathrm{H}_{59} \mathrm{NIU}+\mathrm{C}_{6} \mathrm{H}_{14}\right)$

$944.93 \mathrm{~g} / \mathrm{mol}$

141(2) K

$0.71073 \AA$

Monoclinic

$\mathrm{P} 2{ }_{1} / \mathrm{m}$

$a=11.4776(9) \AA$

$\alpha=90^{\circ}$

$b=13.8658(11) \AA$

$\beta=103.987(1)^{\circ}$

$c=13.1682(10) \AA$

$\gamma=90^{\circ}$

2033.5(3) $\AA^{3}$

2

$1.498 \mathrm{Mg} / \mathrm{m}^{3}$

$1.543 \mathrm{Mg} / \mathrm{m}^{3}$

942

$0.24 \times 0.12 \times 0.04 \mathrm{~mm}^{3}$

1.59 to $26.99^{\circ}$.

$-14<=\mathrm{h}<=14,-17<=\mathrm{k}<=17,-16<=\mathrm{l}<=16$

21556

$4622[R($ int $)=0.0741]$

$100.0 \%$

0.8319 and 0.3935

Full-matrix least-squares on $\mathrm{F}^{2}$

4622 / 0 / 208

0.999

$R 1=0.0358, w R 2=0.0857$

$R 1=0.0462, w R 2=0.0901$

1.616 and -1.452 e. $\AA^{-3}$ 
Table S2. Bond Lengths $[\AA]$ and Angles $\left[{ }^{\circ}\right]$ for $\left(\mathrm{C}_{5} \mathrm{Me}_{5}\right)_{2} \mathrm{U}\left(=\mathrm{N}-2,4,6-{ }^{t} \mathrm{Bu}_{3}-\mathrm{C}_{6} \mathrm{H}_{2}\right)(\mathrm{I})(3)$.

\begin{tabular}{|c|c|c|c|}
\hline $\mathrm{U}(1)-\mathrm{N}(1)$ & $1.975(6)$ & $\mathrm{C}(17)-\mathrm{C}(18) \# 1$ & $1.539(7)$ \\
\hline $\mathrm{U}(1)-\mathrm{C}(5)$ & $2.750(5)$ & $\mathrm{C}(20)-\mathrm{C}(21) \# 1$ & $1.535(7)$ \\
\hline $\mathrm{U}(1)-\mathrm{C}(5) \# 1$ & $2.750(5)$ & $\mathrm{C}(20)-\mathrm{C}(21)$ & $1.535(7)$ \\
\hline $\mathrm{U}(1)-\mathrm{C}(1) \# 1$ & $2.765(5)$ & $C(20)-C(22)$ & $1.547(10)$ \\
\hline $\mathrm{U}(1)-\mathrm{C}(1)$ & $2.765(5)$ & $C(23)-C(25)$ & $1.530(6)$ \\
\hline $\mathrm{U}(1)-\mathrm{C}(4)$ & $2.796(5)$ & $\mathrm{C}(23)-\mathrm{C}(25) \# 1$ & $1.530(6)$ \\
\hline $\mathrm{U}(1)-\mathrm{C}(4) \# 1$ & $2.796(5)$ & $C(23)-C(24)$ & $1.535(9)$ \\
\hline $\mathrm{U}(1)-\mathrm{C}(2)$ & $2.833(5)$ & & \\
\hline $\mathrm{U}(1)-\mathrm{C}(2) \# 1$ & $2.833(5)$ & $\mathrm{N}(1)-\mathrm{U}(1)-\mathrm{C}(5)$ & $87.36(12)$ \\
\hline $\mathrm{U}(1)-\mathrm{C}(3)$ & $2.842(5)$ & $\mathrm{N}(1)-\mathrm{U}(1)-\mathrm{C}(5) \# 1$ & $87.36(12)$ \\
\hline $\mathrm{U}(1)-\mathrm{C}(3) \# 1$ & $2.842(5)$ & $\mathrm{C}(5)-\mathrm{U}(1)-\mathrm{C}(5) \# 1$ & $149.9(2)$ \\
\hline $\mathrm{U}(1)-\mathrm{I}(1)$ & $3.0116(6)$ & $\mathrm{N}(1)-\mathrm{U}(1)-\mathrm{C}(1) \# 1$ & $98.74(12)$ \\
\hline $\mathrm{N}(1)-\mathrm{C}(11)$ & $1.418(8)$ & $\mathrm{C}(5)-\mathrm{U}(1)-\mathrm{C}(1) \# 1$ & $173.59(15)$ \\
\hline $\mathrm{C}(1)-\mathrm{C}(2)$ & $1.408(8)$ & $\mathrm{C}(5) \# 1-\mathrm{U}(1)-\mathrm{C}(1) \# 1$ & $29.79(16)$ \\
\hline $\mathrm{C}(1)-\mathrm{C}(5)$ & $1.418(8)$ & $\mathrm{N}(1)-\mathrm{U}(1)-\mathrm{C}(1)$ & $98.74(12)$ \\
\hline$C(1)-C(6)$ & $1.507(8)$ & $\mathrm{C}(5)-\mathrm{U}(1)-\mathrm{C}(1)$ & $29.79(16)$ \\
\hline $\mathrm{C}(2)-\mathrm{C}(3)$ & $1.403(8)$ & $\mathrm{C}(5) \# 1-\mathrm{U}(1)-\mathrm{C}(1)$ & $173.59(15)$ \\
\hline$C(2)-C(7)$ & $1.517(8)$ & $\mathrm{C}(1) \# 1-\mathrm{U}(1)-\mathrm{C}(1)$ & $149.1(2)$ \\
\hline $\mathrm{C}(3)-\mathrm{C}(4)$ & $1.396(7)$ & $\mathrm{N}(1)-\mathrm{U}(1)-\mathrm{C}(4)$ & $107.86(15)$ \\
\hline $\mathrm{C}(3)-\mathrm{C}(8)$ & $1.506(8)$ & $\mathrm{C}(5)-\mathrm{U}(1)-\mathrm{C}(4)$ & $29.60(15)$ \\
\hline $\mathrm{C}(4)-\mathrm{C}(5)$ & $1.417(7)$ & $\mathrm{C}(5) \# 1-\mathrm{U}(1)-\mathrm{C}(4)$ & $127.51(17)$ \\
\hline$C(4)-C(9)$ & $1.509(8)$ & $\mathrm{C}(1) \# 1-\mathrm{U}(1)-\mathrm{C}(4)$ & $144.60(16)$ \\
\hline$C(5)-C(10)$ & $1.518(7)$ & $\mathrm{C}(1)-\mathrm{U}(1)-\mathrm{C}(4)$ & $48.68(16)$ \\
\hline$C(11)-C(16)$ & $1.436(9)$ & $\mathrm{N}(1)-\mathrm{U}(1)-\mathrm{C}(4) \# 1$ & $107.86(15)$ \\
\hline $\mathrm{C}(11)-\mathrm{C}(12)$ & $1.442(9)$ & $\mathrm{C}(5)-\mathrm{U}(1)-\mathrm{C}(4) \# 1$ & $127.51(17)$ \\
\hline$C(12)-C(13)$ & $1.400(10)$ & $\mathrm{C}(5) \# 1-\mathrm{U}(1)-\mathrm{C}(4) \# 1$ & $29.60(15)$ \\
\hline$C(12)-C(17)$ & $1.532(10)$ & $\mathrm{C}(1) \# 1-\mathrm{U}(1)-\mathrm{C}(4) \# 1$ & $48.68(16)$ \\
\hline$C(13)-C(14)$ & $1.362(10)$ & $\mathrm{C}(1)-\mathrm{U}(1)-\mathrm{C}(4) \# 1$ & $144.60(16)$ \\
\hline$C(14)-C(15)$ & $1.385(10)$ & $\mathrm{C}(4)-\mathrm{U}(1)-\mathrm{C}(4) \# 1$ & $99.9(2)$ \\
\hline$C(14)-C(20)$ & $1.548(9)$ & $\mathrm{N}(1)-\mathrm{U}(1)-\mathrm{C}(2)$ & $127.83(12)$ \\
\hline$C(15)-C(16)$ & $1.392(9)$ & $\mathrm{C}(5)-\mathrm{U}(1)-\mathrm{C}(2)$ & $48.08(15)$ \\
\hline$C(16)-C(23)$ & $1.555(9)$ & $\mathrm{C}(5) \# 1-\mathrm{U}(1)-\mathrm{C}(2)$ & $144.78(16)$ \\
\hline $\mathrm{C}(17)-\mathrm{C}(19)$ & $1.527(11)$ & $\mathrm{C}(1) \# 1-\mathrm{U}(1)-\mathrm{C}(2)$ & $127.45(17)$ \\
\hline $\mathrm{C}(17)-\mathrm{C}(18)$ & $1.539(7)$ & $\mathrm{C}(1)-\mathrm{U}(1)-\mathrm{C}(2)$ & $29.09(16)$ \\
\hline $\mathrm{C}(4)-\mathrm{U}(1)-\mathrm{C}(2)$ & 47.61(16) & $\mathrm{C}(4) \# 1-\mathrm{U}(1)-\mathrm{I}(1)$ & $121.52(11)$ \\
\hline
\end{tabular}




\begin{tabular}{|c|c|c|c|}
\hline $\mathrm{C}(4) \# 1-\mathrm{U}(1)-\mathrm{C}(2)$ & $120.18(17)$ & $\mathrm{C}(2)-\mathrm{U}(1)-\mathrm{I}(1)$ & $75.14(12)$ \\
\hline $\mathrm{N}(1)-\mathrm{U}(1)-\mathrm{C}(2) \# 1$ & $127.83(12)$ & $\mathrm{C}(2) \# 1-\mathrm{U}(1)-\mathrm{I}(1)$ & $75.14(12)$ \\
\hline $\mathrm{C}(5)-\mathrm{U}(1)-\mathrm{C}(2) \# 1$ & $144.78(16)$ & $\mathrm{C}(3)-\mathrm{U}(1)-\mathrm{I}(1)$ & $101.56(12)$ \\
\hline $\mathrm{C}(5) \# 1-\mathrm{U}(1)-\mathrm{C}(2) \# 1$ & $48.08(16)$ & $\mathrm{C}(3) \# 1-\mathrm{U}(1)-\mathrm{I}(1)$ & $101.56(12)$ \\
\hline $\mathrm{C}(1) \# 1-\mathrm{U}(1)-\mathrm{C}(2) \# 1$ & $29.09(16)$ & $\mathrm{C}(11)-\mathrm{N}(1)-\mathrm{U}(1)$ & $169.7(5)$ \\
\hline $\mathrm{C}(1)-\mathrm{U}(1)-\mathrm{C}(2) \# 1$ & $127.45(16)$ & $C(2)-C(1)-C(5)$ & $107.3(5)$ \\
\hline $\mathrm{C}(4)-\mathrm{U}(1)-\mathrm{C}(2) \# 1$ & $120.18(17)$ & $C(2)-C(1)-C(6)$ & $126.9(5)$ \\
\hline $\mathrm{C}(4) \# 1-\mathrm{U}(1)-\mathrm{C}(2) \# 1$ & 47.61(16) & $C(5)-C(1)-C(6)$ & $124.5(5)$ \\
\hline $\mathrm{C}(2)-\mathrm{U}(1)-\mathrm{C}(2) \# 1$ & $100.5(2)$ & $\mathrm{C}(2)-\mathrm{C}(1)-\mathrm{U}(1)$ & $78.1(3)$ \\
\hline N(1)-U(1)-C(3) & $134.59(13)$ & $\mathrm{C}(5)-\mathrm{C}(1)-\mathrm{U}(1)$ & $74.5(3)$ \\
\hline C(5)-U(1)-C(3) & $47.96(15)$ & $\mathrm{C}(6)-\mathrm{C}(1)-\mathrm{U}(1)$ & $123.4(4)$ \\
\hline C(5)\#1-U(1)-C(3) & $125.86(16)$ & $\mathrm{C}(3)-\mathrm{C}(2)-\mathrm{C}(1)$ & $108.5(5)$ \\
\hline $\mathrm{C}(1) \# 1-\mathrm{U}(1)-\mathrm{C}(3)$ & $125.70(16)$ & $C(3)-C(2)-C(7)$ & $125.5(6)$ \\
\hline $\mathrm{C}(1)-\mathrm{U}(1)-\mathrm{C}(3)$ & $47.98(16)$ & $C(1)-C(2)-C(7)$ & $125.3(6)$ \\
\hline $\mathrm{C}(4)-\mathrm{U}(1)-\mathrm{C}(3)$ & $28.67(15)$ & $\mathrm{C}(3)-\mathrm{C}(2)-\mathrm{U}(1)$ & $76.0(3)$ \\
\hline $\mathrm{C}(4) \# 1-\mathrm{U}(1)-\mathrm{C}(3)$ & $96.76(16)$ & $\mathrm{C}(1)-\mathrm{C}(2)-\mathrm{U}(1)$ & $72.8(3)$ \\
\hline $\mathrm{C}(2)-\mathrm{U}(1)-\mathrm{C}(3)$ & $28.63(16)$ & $\mathrm{C}(7)-\mathrm{C}(2)-\mathrm{U}(1)$ & $125.1(4)$ \\
\hline $\mathrm{C}(2) \# 1-\mathrm{U}(1)-\mathrm{C}(3)$ & $97.00(16)$ & $\mathrm{C}(4)-\mathrm{C}(3)-\mathrm{C}(2)$ & $108.5(5)$ \\
\hline $\mathrm{N}(1)-\mathrm{U}(1)-\mathrm{C}(3) \# 1$ & $134.59(12)$ & $\mathrm{C}(4)-\mathrm{C}(3)-\mathrm{C}(8)$ & $124.6(6)$ \\
\hline $\mathrm{C}(5)-\mathrm{U}(1)-\mathrm{C}(3) \# 1$ & $125.86(16)$ & $C(2)-C(3)-C(8)$ & $124.8(6)$ \\
\hline $\mathrm{C}(5) \# 1-\mathrm{U}(1)-\mathrm{C}(3) \# 1$ & $47.96(15)$ & $\mathrm{C}(4)-\mathrm{C}(3)-\mathrm{U}(1)$ & $73.9(3)$ \\
\hline $\mathrm{C}(1) \# 1-\mathrm{U}(1)-\mathrm{C}(3) \# 1$ & $47.98(16)$ & $\mathrm{C}(2)-\mathrm{C}(3)-\mathrm{U}(1)$ & $75.3(3)$ \\
\hline $\mathrm{C}(1)-\mathrm{U}(1)-\mathrm{C}(3) \# 1$ & $125.70(16)$ & $\mathrm{C}(8)-\mathrm{C}(3)-\mathrm{U}(1)$ & $130.2(4)$ \\
\hline $\mathrm{C}(4)-\mathrm{U}(1)-\mathrm{C}(3) \# 1$ & $96.76(16)$ & $C(3)-C(4)-C(5)$ & $107.8(5)$ \\
\hline $\mathrm{C}(4) \# 1-\mathrm{U}(1)-\mathrm{C}(3) \# 1$ & $28.67(15)$ & $\mathrm{C}(3)-\mathrm{C}(4)-\mathrm{C}(9)$ & $124.8(5)$ \\
\hline $\mathrm{C}(2)-\mathrm{U}(1)-\mathrm{C}(3) \# 1$ & $97.00(16)$ & $\mathrm{C}(5)-\mathrm{C}(4)-\mathrm{C}(9)$ & $125.9(5)$ \\
\hline $\mathrm{C}(2) \# 1-\mathrm{U}(1)-\mathrm{C}(3) \# 1$ & $28.63(16)$ & $\mathrm{C}(3)-\mathrm{C}(4)-\mathrm{U}(1)$ & $77.5(3)$ \\
\hline $\mathrm{C}(3)-\mathrm{U}(1)-\mathrm{C}(3) \# 1$ & 81.1(2) & $\mathrm{C}(5)-\mathrm{C}(4)-\mathrm{U}(1)$ & $73.4(3)$ \\
\hline $\mathrm{N}(1)-\mathrm{U}(1)-\mathrm{I}(1)$ & $97.20(16)$ & $C(9)-C(4)-U(1)$ & $126.2(4)$ \\
\hline $\mathrm{C}(5)-\mathrm{U}(1)-\mathrm{I}(1)$ & $105.01(12)$ & $\mathrm{C}(4)-\mathrm{C}(5)-\mathrm{C}(1)$ & 107.9(5) \\
\hline C(5)\#1-U(1)-I(1) & $105.01(12)$ & $C(4)-C(5)-C(10)$ & $127.5(5)$ \\
\hline C(1)\#1-U(1)-I(1) & $76.32(12)$ & $C(1)-C(5)-C(10)$ & $124.1(5)$ \\
\hline $\mathrm{C}(1)-\mathrm{U}(1)-\mathrm{I}(1)$ & $76.32(12)$ & $C(4)-C(5)-U(1)$ & $77.0(3)$ \\
\hline $\mathrm{C}(4)-\mathrm{U}(1)-\mathrm{I}(1)$ & $121.52(11)$ & $C(1)-C(5)-U(1)$ & $75.7(3)$ \\
\hline $\mathrm{C}(10)-\mathrm{C}(5)-\mathrm{U}(1)$ & $119.9(3)$ & $\mathrm{C}(12)-\mathrm{C}(17)-\mathrm{C}(18)$ & 109.3(4) \\
\hline $\mathrm{N}(1)-\mathrm{C}(11)-\mathrm{C}(16)$ & $121.5(6)$ & $\mathrm{C}(19)-\mathrm{C}(17)-\mathrm{C}(18) \# 1$ & $106.6(4)$ \\
\hline
\end{tabular}




$\begin{array}{llll}\mathrm{N}(1)-\mathrm{C}(11)-\mathrm{C}(12) & 121.3(6) & \mathrm{C}(12)-\mathrm{C}(17)-\mathrm{C}(18) \# 1 & 109.3(4) \\ \mathrm{C}(16)-\mathrm{C}(11)-\mathrm{C}(12) & 117.2(6) & \mathrm{C}(18)-\mathrm{C}(17)-\mathrm{C}(18) \# 1 & 112.8(6) \\ \mathrm{C}(13)-\mathrm{C}(12)-\mathrm{C}(11) & 118.9(6) & \mathrm{C}(21) \# 1-\mathrm{C}(20)-\mathrm{C}(21) & 109.8(6) \\ \mathrm{C}(13)-\mathrm{C}(12)-\mathrm{C}(17) & 118.1(6) & \mathrm{C}(21) \# 1-\mathrm{C}(20)-\mathrm{C}(22) & 107.4(4) \\ \mathrm{C}(11)-\mathrm{C}(12)-\mathrm{C}(17) & 123.0(6) & \mathrm{C}(21)-\mathrm{C}(20)-\mathrm{C}(22) & 107.4(4) \\ \mathrm{C}(14)-\mathrm{C}(13)-\mathrm{C}(12) & 124.2(6) & \mathrm{C}(21) \# 1-\mathrm{C}(20)-\mathrm{C}(14) & 109.8(4) \\ \mathrm{C}(13)-\mathrm{C}(14)-\mathrm{C}(15) & 116.6(6) & \mathrm{C}(21)-\mathrm{C}(20)-\mathrm{C}(14) & 109.8(4) \\ \mathrm{C}(13)-\mathrm{C}(14)-\mathrm{C}(20) & 119.0(6) & \mathrm{C}(22)-\mathrm{C}(20)-\mathrm{C}(14) & 112.7(6) \\ \mathrm{C}(15)-\mathrm{C}(14)-\mathrm{C}(20) & 124.4(6) & \mathrm{C}(25)-\mathrm{C}(23)-\mathrm{C}(25) \# 1 & 108.7(6) \\ \mathrm{C}(14)-\mathrm{C}(15)-\mathrm{C}(16) & 124.1(7) & \mathrm{C}(25)-\mathrm{C}(23)-\mathrm{C}(24) & 106.4(4) \\ \mathrm{C}(15)-\mathrm{C}(16)-\mathrm{C}(11) & 119.0(6) & \mathrm{C}(25) \# 1-\mathrm{C}(23)-\mathrm{C}(24) & 106.4(4) \\ \mathrm{C}(15)-\mathrm{C}(16)-\mathrm{C}(23) & 113.8(6) & \mathrm{C}(25)-\mathrm{C}(23)-\mathrm{C}(16) & 110.1(4) \\ \mathrm{C}(11)-\mathrm{C}(16)-\mathrm{C}(23) & 127.2(6) & \mathrm{C}(25) \# 1-\mathrm{C}(23)-\mathrm{C}(16) & 110.1(4) \\ \mathrm{C}(19)-\mathrm{C}(17)-\mathrm{C}(12) & 112.2(6) & \mathrm{C}(24)-\mathrm{C}(23)-\mathrm{C}(16) & 114.8(5) \\ \mathrm{C}(19)-\mathrm{C}(17)-\mathrm{C}(18) & 106.6(4) & & \end{array}$


Figure S2. Molecular structure of complex 4 with thermal ellipsoids at the $50 \%$ probability level. Selected bond distances $(\AA)$ and angles $\left({ }^{\circ}\right)$ for 4: $\mathrm{U}(1)-\mathrm{I}(1) 3.03857, \mathrm{U}(1)-\mathrm{N}(1)$ 1.974(7), N(1)-C(21) 1.406(10), $\mathrm{U}(1)-\mathrm{C}_{5} \mathrm{Me}_{5 \text { (cent) }}$ 2.454, 2.459, N(1)-U(1)-I(1) 106.6(2), C(21)-N(1)-U(1) 170.7(6), $\mathrm{C}_{5} \mathrm{Me}_{5(\mathrm{cent})}-\mathrm{U}(1)-\mathrm{C}_{5} \mathrm{Me}_{5(\mathrm{cent})}$ 134.07. Selected bond distances $(\AA)$ and angles ( $\left.{ }^{\circ}\right)$ for 4': U(2)-I(2) 3.0482(7), U(2)-N(2) 1.960(7), N(2)-C(53) 1.431(10), U(2)-C $\mathrm{C}_{5} \mathrm{Me}_{5(\text { cent })}$ 2.464, 2.459, N(2)-U(2)-I(2) 107.0(2), C(53)-N(2)-U(2) 165.9(6), $\mathrm{C}_{5} \mathrm{Me}_{5(\mathrm{cent})}-\mathrm{U}(2)-\mathrm{C}_{5} \mathrm{Me}_{5 \text { (cent) }} 133.98$.

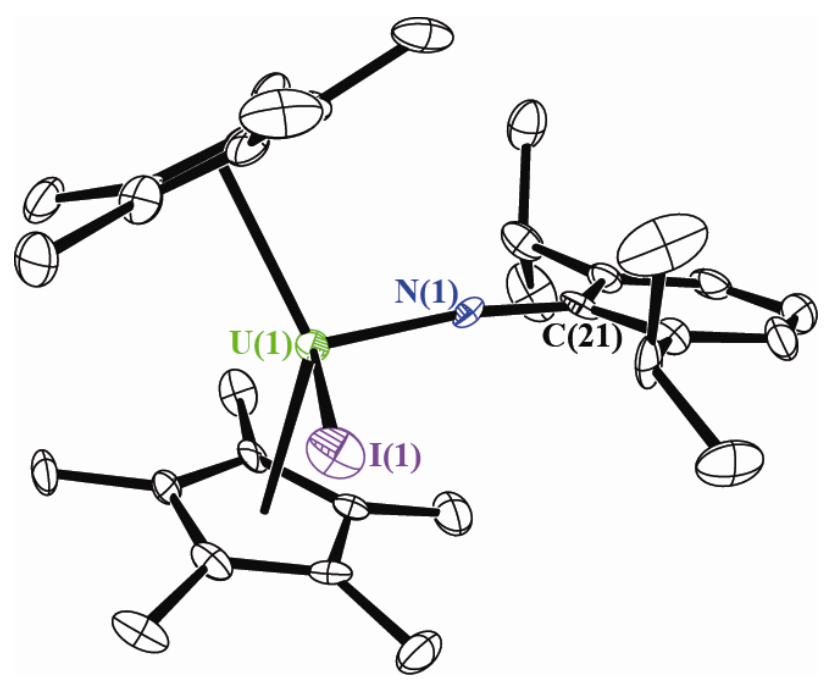


Table S3. Crystal Data and Structure Refinement for $\left(\mathrm{C}_{5} \mathrm{Me}_{5}\right)_{2} \mathrm{U}\left(=\mathrm{N}-2,6-{ }^{i} \mathrm{Pr}_{2}-\mathrm{C}_{6} \mathrm{H}_{3}\right)(\mathrm{I})$ (4).

Empirical formula

Formula weight

Temperature

Wavelength

Crystal system

Space group

Unit cell dimensions

Volume

Z

Density (calculated)

Absorption coefficient

$\mathrm{F}(000)$

Crystal size

Theta range for data collection

Index ranges

Reflections collected

Independent reflections

Completeness to theta $=25.00^{\circ}$

Absorption correction

Max. and min. transmission

Refinement method

Data / restraints / parameters

Goodness-of-fit on F2

Final $\mathrm{R}$ indices $[\mathrm{I}>2 \operatorname{sigma}(\mathrm{I})]$

$\mathrm{R}$ indices (all data)

Absolute structure parameter

Largest diff. peak and hole
$\mathrm{C}_{32} \mathrm{H}_{47} \mathrm{INU}$

$810.64 \mathrm{~g} / \mathrm{mol}$

141(2) K

$0.71073 \AA$

Orthorhombic

$\mathrm{P} 2{ }_{1} 2_{1} 2_{1}$

$a=16.5588(9) \AA$

$\alpha=90^{\circ}$

$b=18.7054(10) \AA$

$\beta=90^{\circ}$

$c=19.5958(10) \AA$

$\gamma=90^{\circ}$

6069.6(6) $\AA^{3}$

8

$1.774 \mathrm{Mg} / \mathrm{m}^{3}$

$6.386 \mathrm{~mm}^{-1}$

3128

$0.20 \times 0.08 \times 0.04 \mathrm{~mm}^{3}$

1.50 to $25.36^{\circ}$.

$-19<=\mathrm{h}<=19,-22<=\mathrm{k}<=22,-23<=\mathrm{l}<=23$

61796

$11113[R($ int $)=0.0874]$

$99.9 \%$

Semi-empirical from equivalents

0.7843 and 0.3616

Full-matrix least-squares on $\mathrm{F}^{2}$

$11113 / 12$ / 659

1.023

$R 1=0.0373, w R 2=0.0662$

$R 1=0.0490, w R 2=0.0708$

$0.529(5)$

0.814 and -0.682 e. $\AA^{-3}$ 
Table S4. Bond Lengths $[\AA]$ and Angles $\left[^{\circ}\right]$ for $\left(\mathrm{C}_{5} \mathrm{Me}_{5}\right)_{2} \mathrm{U}\left(=\mathrm{N}-2,6{ }^{i} \mathrm{Pr}_{2}-\mathrm{C}_{6} \mathrm{H}_{3}\right)(\mathrm{I})$ (4)

\begin{tabular}{|c|c|c|c|}
\hline $\mathrm{U}(1)-\mathrm{N}(1)$ & $1.974(7)$ & $\mathrm{C}(4)-\mathrm{C}(9)$ & $1.495(11)$ \\
\hline $\mathrm{U}(1)-\mathrm{C}(11)$ & $2.701(9)$ & $C(5)-C(10)$ & $1.502(12)$ \\
\hline $\mathrm{U}(1)-\mathrm{C}(1)$ & $2.702(7)$ & $\mathrm{C}(11)-\mathrm{C}(12)$ & $1.390(12)$ \\
\hline $\mathrm{U}(1)-\mathrm{C}(5)$ & $2.709(8)$ & $C(11)-C(15)$ & $1.413(12)$ \\
\hline $\mathrm{U}(1)-\mathrm{C}(15)$ & $2.732(8)$ & $\mathrm{C}(11)-\mathrm{C}(16)$ & $1.529(12)$ \\
\hline $\mathrm{U}(1)-\mathrm{C}(4)$ & $2.733(8)$ & $C(12)-C(13)$ & $1.390(12)$ \\
\hline $\mathrm{U}(1)-\mathrm{C}(12)$ & $2.744(8)$ & $C(12)-C(17)$ & $1.511(11)$ \\
\hline $\mathrm{U}(1)-\mathrm{C}(13)$ & $2.747(8)$ & $C(13)-C(14)$ & $1.434(11)$ \\
\hline $\mathrm{U}(1)-\mathrm{C}(2)$ & $2.749(8)$ & $\mathrm{C}(13)-\mathrm{C}(18)$ & $1.534(11)$ \\
\hline $\mathrm{U}(1)-\mathrm{C}(14)$ & $2.754(8)$ & $\mathrm{C}(14)-\mathrm{C}(15)$ & $1.412(12)$ \\
\hline $\mathrm{U}(1)-\mathrm{C}(3)$ & $2.777(8)$ & $C(14)-C(19)$ & $1.515(11)$ \\
\hline $\mathrm{U}(1)-\mathrm{I}(1)$ & $3.0385(7)$ & $C(15)-C(20)$ & $1.503(11)$ \\
\hline $\mathrm{U}(2)-\mathrm{N}(2)$ & $1.960(7)$ & $C(21)-C(26)$ & $1.404(12)$ \\
\hline $\mathrm{U}(2)-\mathrm{C}(44)$ & $2.715(8)$ & $C(21)-C(22)$ & $1.438(12)$ \\
\hline $\mathrm{U}(2)-\mathrm{C}(35)$ & $2.717(9)$ & $C(22)-C(23)$ & $1.379(12)$ \\
\hline $\mathrm{U}(2)-\mathrm{C}(36)$ & $2.720(10)$ & $C(22)-C(27)$ & $1.509(11)$ \\
\hline $\mathrm{U}(2)-\mathrm{C}(43)$ & $2.724(8)$ & $C(23)-C(24)$ & $1.401(13)$ \\
\hline $\mathrm{U}(2)-\mathrm{C}(47)$ & $2.740(8)$ & $C(24)-C(25)$ & $1.373(12)$ \\
\hline $\mathrm{U}(2)-\mathrm{C}(34)$ & $2.744(9)$ & $C(25)-C(26)$ & $1.394(12)$ \\
\hline $\mathrm{U}(2)-\mathrm{C}(33)$ & $2.747(8)$ & $C(26)-C(30)$ & $1.503(11)$ \\
\hline $\mathrm{U}(2)-\mathrm{C}(46)$ & $2.753(9)$ & $\mathrm{C}(27)-\mathrm{C}(29)$ & $1.511(11)$ \\
\hline $\mathrm{U}(2)-\mathrm{C}(37)$ & $2.756(10)$ & $C(27)-C(28)$ & $1.527(12)$ \\
\hline $\mathrm{U}(2)-\mathrm{C}(45)$ & $2.761(9)$ & $\mathrm{C}(30)-\mathrm{C}(31)$ & $1.506(12)$ \\
\hline $\mathrm{U}(2)-\mathrm{I}(2)$ & $3.0482(7)$ & $\mathrm{C}(30)-\mathrm{C}(32)$ & $1.530(12)$ \\
\hline $\mathrm{N}(1)-\mathrm{C}(21)$ & $1.406(10)$ & $\mathrm{C}(33)-\mathrm{C}(37)$ & $1.403(13)$ \\
\hline $\mathrm{N}(2)-\mathrm{C}(53)$ & $1.431(10)$ & $\mathrm{C}(33)-\mathrm{C}(34)$ & $1.408(12)$ \\
\hline$C(1)-C(5)$ & $1.416(11)$ & $\mathrm{C}(33)-\mathrm{C}(38)$ & $1.490(13)$ \\
\hline $\mathrm{C}(1)-\mathrm{C}(2)$ & $1.423(11)$ & $\mathrm{C}(34)-\mathrm{C}(35)$ & $1.432(13)$ \\
\hline$C(1)-C(6)$ & $1.521(11)$ & $\mathrm{C}(34)-\mathrm{C}(39)$ & $1.497(12)$ \\
\hline $\mathrm{C}(2)-\mathrm{C}(3)$ & $1.403(11)$ & $C(35)-C(36)$ & $1.407(13)$ \\
\hline$C(2)-C(7)$ & $1.496(10)$ & $C(35)-C(40)$ & $1.496(13)$ \\
\hline$C(3)-C(4)$ & $1.421(11)$ & $C(36)-C(37)$ & $1.408(13)$ \\
\hline$C(3)-C(8)$ & $1.502(11)$ & $C(36)-C(41)$ & $1.508(12)$ \\
\hline$C(4)-C(5)$ & $1.427(12)$ & $C(37)-C(42)$ & $1.520(13)$ \\
\hline $\mathrm{C}(43)-\mathrm{C}(44)$ & $1.427(12)$ & $\mathrm{C}(5)-\mathrm{U}(1)-\mathrm{C}(4)$ & $30.4(3)$ \\
\hline
\end{tabular}




\begin{tabular}{|c|c|c|c|}
\hline$C(43)-C(47)$ & $1.447(13)$ & $\mathrm{C}(15)-\mathrm{U}(1)-\mathrm{C}(4)$ & $156.2(3)$ \\
\hline $\mathrm{C}(43)-\mathrm{C}(48)$ & $1.485(12)$ & $\mathrm{N}(1)-\mathrm{U}(1)-\mathrm{C}(12)$ & $119.5(3)$ \\
\hline $\mathrm{C}(44)-\mathrm{C}(45)$ & $1.404(13)$ & $\mathrm{C}(11)-\mathrm{U}(1)-\mathrm{C}(12)$ & $29.6(2)$ \\
\hline C(44)-C(49) & $1.508(12)$ & $\mathrm{C}(1)-\mathrm{U}(1)-\mathrm{C}(12)$ & $91.7(2)$ \\
\hline$C(45)-C(46)$ & $1.375(12)$ & $\mathrm{C}(5)-\mathrm{U}(1)-\mathrm{C}(12)$ & $121.7(3)$ \\
\hline $\mathrm{C}(45)-\mathrm{C}(50)$ & $1.510(12)$ & $\mathrm{C}(15)-\mathrm{U}(1)-\mathrm{C}(12)$ & $49.0(2)$ \\
\hline$C(46)-C(47)$ & $1.375(12)$ & $\mathrm{C}(4)-\mathrm{U}(1)-\mathrm{C}(12)$ & $133.3(3)$ \\
\hline $\mathrm{C}(46)-\mathrm{C}(51)$ & $1.541(12)$ & $\mathrm{N}(1)-\mathrm{U}(1)-\mathrm{C}(13)$ & $90.2(3)$ \\
\hline $\mathrm{C}(47)-\mathrm{C}(52)$ & $1.517(12)$ & $\mathrm{C}(11)-\mathrm{U}(1)-\mathrm{C}(13)$ & $49.2(3)$ \\
\hline $\mathrm{C}(53)-\mathrm{C}(58)$ & $1.386(12)$ & $\mathrm{C}(1)-\mathrm{U}(1)-\mathrm{C}(13)$ & $97.9(2)$ \\
\hline $\mathrm{C}(53)-\mathrm{C}(54)$ & $1.440(12)$ & $\mathrm{C}(5)-\mathrm{U}(1)-\mathrm{C}(13)$ & $121.2(3)$ \\
\hline$C(54)-C(55)$ & $1.360(11)$ & $C(15)-U(1)-C(13)$ & $49.5(2)$ \\
\hline$C(54)-C(59)$ & $1.507(11)$ & $\mathrm{C}(4)-\mathrm{U}(1)-\mathrm{C}(13)$ & $147.8(3)$ \\
\hline$C(55)-C(56)$ & $1.373(12)$ & $C(12)-U(1)-C(13)$ & $29.3(2)$ \\
\hline$C(56)-C(57)$ & $1.378(12)$ & $\mathrm{N}(1)-\mathrm{U}(1)-\mathrm{C}(2)$ & $125.0(3)$ \\
\hline $\mathrm{C}(57)-\mathrm{C}(58)$ & $1.389(11)$ & $\mathrm{C}(11)-\mathrm{U}(1)-\mathrm{C}(2)$ & $95.1(3)$ \\
\hline$C(58)-C(62)$ & $1.510(11)$ & $\mathrm{C}(1)-\mathrm{U}(1)-\mathrm{C}(2)$ & $30.3(2)$ \\
\hline $\mathrm{C}(59)-\mathrm{C}(60)$ & $1.536(12)$ & $\mathrm{C}(5)-\mathrm{U}(1)-\mathrm{C}(2)$ & $49.9(2)$ \\
\hline $\mathrm{C}(59)-\mathrm{C}(61)$ & $1.542(12)$ & $\mathrm{C}(15)-\mathrm{U}(1)-\mathrm{C}(2)$ & $125.2(2)$ \\
\hline$C(62)-C(64)$ & $1.522(11)$ & $\mathrm{C}(4)-\mathrm{U}(1)-\mathrm{C}(2)$ & $49.5(2)$ \\
\hline \multirow[t]{2}{*}{$C(62)-C(63)$} & $1.528(11)$ & $\mathrm{C}(12)-\mathrm{U}(1)-\mathrm{C}(2)$ & $83.8(2)$ \\
\hline & & $\mathrm{C}(13)-\mathrm{U}(1)-\mathrm{C}(2)$ & $103.6(2)$ \\
\hline $\mathrm{N}(1)-\mathrm{U}(1)-\mathrm{C}(11)$ & $130.6(3)$ & $\mathrm{N}(1)-\mathrm{U}(1)-\mathrm{C}(14)$ & $81.2(3)$ \\
\hline $\mathrm{N}(1)-\mathrm{U}(1)-\mathrm{C}(1)$ & $95.9(3)$ & $\mathrm{C}(11)-\mathrm{U}(1)-\mathrm{C}(14)$ & $49.4(3)$ \\
\hline $\mathrm{C}(11)-\mathrm{U}(1)-\mathrm{C}(1)$ & 114.1(3) & $\mathrm{C}(1)-\mathrm{U}(1)-\mathrm{C}(14)$ & $127.4(2)$ \\
\hline $\mathrm{N}(1)-\mathrm{U}(1)-\mathrm{C}(5)$ & $77.3(3)$ & $\mathrm{C}(5)-\mathrm{U}(1)-\mathrm{C}(14)$ & $144.1(3)$ \\
\hline $\mathrm{C}(11)-\mathrm{U}(1)-\mathrm{C}(5)$ & $143.5(3)$ & $\mathrm{C}(15)-\mathrm{U}(1)-\mathrm{C}(14)$ & $29.8(2)$ \\
\hline $\mathrm{C}(1)-\mathrm{U}(1)-\mathrm{C}(5)$ & $30.3(2)$ & $\mathrm{C}(4)-\mathrm{U}(1)-\mathrm{C}(14)$ & $173.8(3)$ \\
\hline $\mathrm{N}(1)-\mathrm{U}(1)-\mathrm{C}(15)$ & $104.3(3)$ & $C(12)-U(1)-C(14)$ & $48.9(2)$ \\
\hline $\mathrm{C}(11)-\mathrm{U}(1)-\mathrm{C}(15)$ & $30.1(2)$ & $\mathrm{C}(13)-\mathrm{U}(1)-\mathrm{C}(14)$ & $30.2(2)$ \\
\hline $\mathrm{C}(1)-\mathrm{U}(1)-\mathrm{C}(15)$ & $140.7(2)$ & $C(2)-U(1)-C(14)$ & $131.9(2)$ \\
\hline $\mathrm{C}(5)-\mathrm{U}(1)-\mathrm{C}(15)$ & $170.2(3)$ & $\mathrm{N}(1)-\mathrm{U}(1)-\mathrm{C}(3)$ & $122.8(3)$ \\
\hline $\mathrm{N}(1)-\mathrm{U}(1)-\mathrm{C}(4)$ & $93.4(3)$ & $\mathrm{C}(11)-\mathrm{U}(1)-\mathrm{C}(3)$ & $106.4(3)$ \\
\hline$C(11)-U(1)-C(4)$ & $136.0(3)$ & $C(1)-U(1)-C(3)$ & $49.2(2)$ \\
\hline$C(1)-U(1)-C(4)$ & $49.9(3)$ & $\mathrm{C}(5)-\mathrm{U}(1)-\mathrm{C}(3)$ & $49.5(2)$ \\
\hline $\mathrm{C}(15)-\mathrm{U}(1)-\mathrm{C}(3)$ & $131.8(3)$ & $\mathrm{C}(43)-\mathrm{U}(2)-\mathrm{C}(34)$ & $132.5(3)$ \\
\hline
\end{tabular}




\begin{tabular}{|c|c|c|c|}
\hline$C(4)-U(1)-C(3)$ & $29.9(2)$ & $\mathrm{C}(47)-\mathrm{U}(2)-\mathrm{C}(34)$ & $127.6(3)$ \\
\hline $\mathrm{C}(12)-\mathrm{U}(1)-\mathrm{C}(3)$ & $107.0(3)$ & $\mathrm{N}(2)-\mathrm{U}(2)-\mathrm{C}(33)$ & $131.6(3)$ \\
\hline $\mathrm{C}(13)-\mathrm{U}(1)-\mathrm{C}(3)$ & $131.6(3)$ & $C(44)-U(2)-C(33)$ & $135.1(3)$ \\
\hline$C(2)-U(1)-C(3)$ & $29.4(2)$ & $\mathrm{C}(35)-\mathrm{U}(2)-\mathrm{C}(33)$ & $49.8(3)$ \\
\hline $\mathrm{C}(14)-\mathrm{U}(1)-\mathrm{C}(3)$ & $154.7(3)$ & $\mathrm{C}(36)-\mathrm{U}(2)-\mathrm{C}(33)$ & $49.4(3)$ \\
\hline $\mathrm{N}(1)-\mathrm{U}(1)-\mathrm{I}(1)$ & $106.6(2)$ & $C(43)-U(2)-C(33)$ & $105.4(3)$ \\
\hline $\mathrm{C}(11)-\mathrm{U}(1)-\mathrm{I}(1)$ & $86.59(19)$ & $\mathrm{C}(47)-\mathrm{U}(2)-\mathrm{C}(33)$ & $98.4(3)$ \\
\hline $\mathrm{C}(1)-\mathrm{U}(1)-\mathrm{I}(1)$ & $126.57(18)$ & $\mathrm{C}(34)-\mathrm{U}(2)-\mathrm{C}(33)$ & $29.7(3)$ \\
\hline $\mathrm{C}(5)-\mathrm{U}(1)-\mathrm{I}(1)$ & $109.6(2)$ & $\mathrm{N}(2)-\mathrm{U}(2)-\mathrm{C}(46)$ & $108.6(3)$ \\
\hline $\mathrm{C}(15)-\mathrm{U}(1)-\mathrm{I}(1)$ & $79.42(19)$ & $\mathrm{C}(44)-\mathrm{U}(2)-\mathrm{C}(46)$ & $48.4(3)$ \\
\hline $\mathrm{C}(4)-\mathrm{U}(1)-\mathrm{I}(1)$ & $80.4(2)$ & $C(35)-U(2)-C(46)$ & $167.9(3)$ \\
\hline $\mathrm{C}(12)-\mathrm{U}(1)-\mathrm{I}(1)$ & $115.89(19)$ & $\mathrm{C}(36)-\mathrm{U}(2)-\mathrm{C}(46)$ & $140.6(3)$ \\
\hline $\mathrm{C}(13)-\mathrm{U}(1)-\mathrm{I}(1)$ & $128.91(17)$ & $\mathrm{C}(43)-\mathrm{U}(2)-\mathrm{C}(46)$ & $49.3(3)$ \\
\hline $\mathrm{C}(2)-\mathrm{U}(1)-\mathrm{I}(1)$ & $104.67(18)$ & $\mathrm{C}(47)-\mathrm{U}(2)-\mathrm{C}(46)$ & $29.0(3)$ \\
\hline C(14)-U(1)-I(1) & $103.96(17)$ & $\mathrm{C}(34)-\mathrm{U}(2)-\mathrm{C}(46)$ & $143.4(3)$ \\
\hline $\mathrm{C}(3)-\mathrm{U}(1)-\mathrm{I}(1)$ & $78.32(17)$ & $\mathrm{C}(33)-\mathrm{U}(2)-\mathrm{C}(46)$ & $119.7(3)$ \\
\hline $\mathrm{N}(2)-\mathrm{U}(2)-\mathrm{C}(44)$ & $80.2(3)$ & $\mathrm{N}(2)-\mathrm{U}(2)-\mathrm{C}(37)$ & $124.0(3)$ \\
\hline $\mathrm{N}(2)-\mathrm{U}(2)-\mathrm{C}(35)$ & $82.5(3)$ & $\mathrm{C}(44)-\mathrm{U}(2)-\mathrm{C}(37)$ & $109.9(3)$ \\
\hline $\mathrm{C}(44)-\mathrm{U}(2)-\mathrm{C}(35)$ & $132.3(3)$ & $C(35)-U(2)-C(37)$ & $49.2(3)$ \\
\hline $\mathrm{N}(2)-\mathrm{U}(2)-\mathrm{C}(36)$ & $94.2(3)$ & $\mathrm{C}(36)-\mathrm{U}(2)-\mathrm{C}(37)$ & $29.8(3)$ \\
\hline $\mathrm{C}(44)-\mathrm{U}(2)-\mathrm{C}(36)$ & $108.2(3)$ & $C(43)-U(2)-C(37)$ & $84.1(3)$ \\
\hline $\mathrm{C}(35)-\mathrm{U}(2)-\mathrm{C}(36)$ & $30.0(3)$ & $\mathrm{C}(47)-\mathrm{U}(2)-\mathrm{C}(37)$ & $90.4(3)$ \\
\hline $\mathrm{N}(2)-\mathrm{U}(2)-\mathrm{C}(43)$ & $108.0(3)$ & $\mathrm{C}(34)-\mathrm{U}(2)-\mathrm{C}(37)$ & $48.7(3)$ \\
\hline $\mathrm{C}(44)-\mathrm{U}(2)-\mathrm{C}(43)$ & $30.4(3)$ & $C(33)-U(2)-C(37)$ & $29.5(3)$ \\
\hline $\mathrm{C}(35)-\mathrm{U}(2)-\mathrm{C}(43)$ & $123.6(3)$ & $\mathrm{C}(46)-\mathrm{U}(2)-\mathrm{C}(37)$ & $118.8(3)$ \\
\hline $\mathrm{C}(36)-\mathrm{U}(2)-\mathrm{C}(43)$ & $93.7(3)$ & $\mathrm{N}(2)-\mathrm{U}(2)-\mathrm{C}(45)$ & $81.3(3)$ \\
\hline $\mathrm{N}(2)-\mathrm{U}(2)-\mathrm{C}(47)$ & $127.6(3)$ & $C(44)-U(2)-C(45)$ & $29.7(3)$ \\
\hline $\mathrm{C}(44)-\mathrm{U}(2)-\mathrm{C}(47)$ & $49.4(3)$ & $C(35)-U(2)-C(45)$ & $158.3(3)$ \\
\hline $\mathrm{C}(35)-\mathrm{U}(2)-\mathrm{C}(47)$ & $139.5(3)$ & $C(36)-U(2)-C(45)$ & $137.9(3)$ \\
\hline $\mathrm{C}(36)-\mathrm{U}(2)-\mathrm{C}(47)$ & $112.2(3)$ & $\mathrm{C}(43)-\mathrm{U}(2)-\mathrm{C}(45)$ & $49.7(3)$ \\
\hline $\mathrm{C}(43)-\mathrm{U}(2)-\mathrm{C}(47)$ & $30.7(3)$ & $\mathrm{C}(47)-\mathrm{U}(2)-\mathrm{C}(45)$ & $48.5(3)$ \\
\hline $\mathrm{N}(2)-\mathrm{U}(2)-\mathrm{C}(34)$ & 104.1(3) & $\mathrm{C}(34)-\mathrm{U}(2)-\mathrm{C}(45)$ & $171.3(3)$ \\
\hline $\mathrm{C}(44)-\mathrm{U}(2)-\mathrm{C}(34)$ & $157.0(3)$ & $C(33)-U(2)-C(45)$ & $146.6(3)$ \\
\hline $\mathrm{C}(35)-\mathrm{U}(2)-\mathrm{C}(34)$ & $30.4(3)$ & $\mathrm{C}(46)-\mathrm{U}(2)-\mathrm{C}(45)$ & $28.9(3)$ \\
\hline $\mathrm{C}(36)-\mathrm{U}(2)-\mathrm{C}(34)$ & $49.4(3)$ & $\mathrm{C}(37)-\mathrm{U}(2)-\mathrm{C}(45)$ & $133.6(3)$ \\
\hline $\mathrm{N}(2)-\mathrm{U}(2)-\mathrm{I}(2)$ & $107.0(2)$ & $\mathrm{C}(9)-\mathrm{C}(4)-\mathrm{U}(1)$ & $121.2(5)$ \\
\hline
\end{tabular}




\begin{tabular}{|c|c|c|c|}
\hline $\mathrm{C}(44)-\mathrm{U}(2)-\mathrm{I}(2)$ & $122.8(2)$ & $C(1)-C(5)-C(4)$ & $107.5(8)$ \\
\hline $\mathrm{C}(35)-\mathrm{U}(2)-\mathrm{I}(2)$ & $104.7(2)$ & $C(1)-C(5)-C(10)$ & $127.9(9)$ \\
\hline $\mathrm{C}(36)-\mathrm{U}(2)-\mathrm{I}(2)$ & $127.00(19)$ & $C(4)-C(5)-C(10)$ & $123.7(8)$ \\
\hline $\mathrm{C}(43)-\mathrm{U}(2)-\mathrm{I}(2)$ & $122.7(2)$ & $\mathrm{C}(1)-\mathrm{C}(5)-\mathrm{U}(1)$ & $74.6(4)$ \\
\hline $\mathrm{C}(47)-\mathrm{U}(2)-\mathrm{I}(2)$ & $92.3(2)$ & $C(4)-C(5)-U(1)$ & $75.8(5)$ \\
\hline $\mathrm{C}(34)-\mathrm{U}(2)-\mathrm{I}(2)$ & $78.11(18)$ & $C(10)-C(5)-U(1)$ & $123.8(5)$ \\
\hline $\mathrm{C}(33)-\mathrm{U}(2)-\mathrm{I}(2)$ & $82.14(19)$ & $C(12)-C(11)-C(15)$ & $108.2(8)$ \\
\hline $\mathrm{C}(46)-\mathrm{U}(2)-\mathrm{I}(2)$ & $77.09(18)$ & $C(12)-C(11)-C(16)$ & $125.7(8)$ \\
\hline $\mathrm{C}(37)-\mathrm{U}(2)-\mathrm{I}(2)$ & $110.9(2)$ & $C(15)-C(11)-C(16)$ & $126.0(8)$ \\
\hline $\mathrm{C}(45)-\mathrm{U}(2)-\mathrm{I}(2)$ & $93.8(2)$ & $\mathrm{C}(12)-\mathrm{C}(11)-\mathrm{U}(1)$ & $76.9(5)$ \\
\hline $\mathrm{C}(21)-\mathrm{N}(1)-\mathrm{U}(1)$ & $170.7(6)$ & $C(15)-C(11)-U(1)$ & $76.1(5)$ \\
\hline $\mathrm{C}(53)-\mathrm{N}(2)-\mathrm{U}(2)$ & $165.9(6)$ & $\mathrm{C}(16)-\mathrm{C}(11)-\mathrm{U}(1)$ & $115.2(6)$ \\
\hline$C(5)-C(1)-C(2)$ & $108.4(8)$ & $\mathrm{C}(11)-\mathrm{C}(12)-\mathrm{C}(13)$ & $109.4(8)$ \\
\hline$C(5)-C(1)-C(6)$ & $125.9(8)$ & $\mathrm{C}(11)-\mathrm{C}(12)-\mathrm{C}(17)$ & $125.8(9)$ \\
\hline$C(2)-C(1)-C(6)$ & $125.4(7)$ & $\mathrm{C}(13)-\mathrm{C}(12)-\mathrm{C}(17)$ & $123.4(9)$ \\
\hline$C(5)-C(1)-U(1)$ & $75.1(4)$ & $\mathrm{C}(11)-\mathrm{C}(12)-\mathrm{U}(1)$ & $73.5(5)$ \\
\hline$C(2)-C(1)-U(1)$ & $76.7(4)$ & $\mathrm{C}(13)-\mathrm{C}(12)-\mathrm{U}(1)$ & $75.4(5)$ \\
\hline$C(6)-C(1)-U(1)$ & $119.3(5)$ & $\mathrm{C}(17)-\mathrm{C}(12)-\mathrm{U}(1)$ & $128.5(6)$ \\
\hline$C(3)-C(2)-C(1)$ & $107.7(7)$ & $C(12)-C(13)-C(14)$ & $107.3(8)$ \\
\hline$C(3)-C(2)-C(7)$ & $123.5(8)$ & $C(12)-C(13)-C(18)$ & $127.3(8)$ \\
\hline$C(1)-C(2)-C(7)$ & $127.2(8)$ & $C(14)-C(13)-C(18)$ & $124.6(7)$ \\
\hline$C(3)-C(2)-U(1)$ & $76.4(5)$ & $\mathrm{C}(12)-\mathrm{C}(13)-\mathrm{U}(1)$ & $75.2(5)$ \\
\hline$C(1)-C(2)-U(1)$ & $73.0(4)$ & $\mathrm{C}(14)-\mathrm{C}(13)-\mathrm{U}(1)$ & $75.2(5)$ \\
\hline$C(7)-C(2)-U(1)$ & $127.6(5)$ & $\mathrm{C}(18)-\mathrm{C}(13)-\mathrm{U}(1)$ & $123.7(5)$ \\
\hline$C(2)-C(3)-C(4)$ & $108.7(7)$ & $C(15)-C(14)-C(13)$ & $107.5(7)$ \\
\hline$C(2)-C(3)-C(8)$ & $124.8(8)$ & $C(15)-C(14)-C(19)$ & $127.0(8)$ \\
\hline $\mathrm{C}(4)-\mathrm{C}(3)-\mathrm{C}(8)$ & $126.1(8)$ & $\mathrm{C}(13)-\mathrm{C}(14)-\mathrm{C}(19)$ & $125.2(7)$ \\
\hline$C(2)-C(3)-U(1)$ & $74.2(5)$ & $\mathrm{C}(15)-\mathrm{C}(14)-\mathrm{U}(1)$ & $74.2(5)$ \\
\hline$C(4)-C(3)-U(1)$ & $73.3(5)$ & $C(13)-C(14)-U(1)$ & $74.6(5)$ \\
\hline $\mathrm{C}(8)-\mathrm{C}(3)-\mathrm{U}(1)$ & $124.0(5)$ & $\mathrm{C}(19)-\mathrm{C}(14)-\mathrm{U}(1)$ & $122.3(6)$ \\
\hline$C(3)-C(4)-C(5)$ & 107.6(7) & $C(14)-C(15)-C(11)$ & $107.6(8)$ \\
\hline$C(3)-C(4)-C(9)$ & $129.1(8)$ & $C(14)-C(15)-C(20)$ & $124.0(8)$ \\
\hline$C(5)-C(4)-C(9)$ & $122.8(8)$ & $C(11)-C(15)-C(20)$ & $127.5(8)$ \\
\hline$C(3)-C(4)-U(1)$ & $76.8(5)$ & $\mathrm{C}(14)-\mathrm{C}(15)-\mathrm{U}(1)$ & $75.9(5)$ \\
\hline$C(5)-C(4)-U(1)$ & $73.8(5)$ & $\mathrm{C}(11)-\mathrm{C}(15)-\mathrm{U}(1)$ & $73.7(5)$ \\
\hline$C(20)-C(15)-U(1)$ & $124.9(6)$ & $\mathrm{C}(40)-\mathrm{C}(35)-\mathrm{U}(2)$ & $123.6(6)$ \\
\hline
\end{tabular}




\begin{tabular}{|c|c|c|c|}
\hline $\mathrm{C}(26)-\mathrm{C}(21)-\mathrm{N}(1)$ & $122.2(8)$ & $\mathrm{C}(35)-\mathrm{C}(36)-\mathrm{C}(37)$ & 108.2(9) \\
\hline$C(26)-C(21)-C(22)$ & $119.8(8)$ & $\mathrm{C}(35)-\mathrm{C}(36)-\mathrm{C}(41)$ & $125.0(10)$ \\
\hline $\mathrm{N}(1)-\mathrm{C}(21)-\mathrm{C}(22)$ & $117.9(8)$ & $\mathrm{C}(37)-\mathrm{C}(36)-\mathrm{C}(41)$ & $126.5(10)$ \\
\hline$C(23)-C(22)-C(21)$ & $118.4(8)$ & $\mathrm{C}(35)-\mathrm{C}(36)-\mathrm{U}(2)$ & $74.9(6)$ \\
\hline$C(23)-C(22)-C(27)$ & $121.4(8)$ & $\mathrm{C}(37)-\mathrm{C}(36)-\mathrm{U}(2)$ & $76.5(6)$ \\
\hline $\mathrm{C}(21)-\mathrm{C}(22)-\mathrm{C}(27)$ & $120.1(8)$ & $\mathrm{C}(41)-\mathrm{C}(36)-\mathrm{U}(2)$ & $119.3(7)$ \\
\hline$C(22)-C(23)-C(24)$ & $121.9(9)$ & $\mathrm{C}(33)-\mathrm{C}(37)-\mathrm{C}(36)$ & $108.8(9)$ \\
\hline$C(25)-C(24)-C(23)$ & 118.7(9) & $\mathrm{C}(33)-\mathrm{C}(37)-\mathrm{C}(42)$ & $124.6(10)$ \\
\hline$C(24)-C(25)-C(26)$ & $122.3(9)$ & $\mathrm{C}(36)-\mathrm{C}(37)-\mathrm{C}(42)$ & $125.4(10)$ \\
\hline$C(25)-C(26)-C(21)$ & $118.7(8)$ & $\mathrm{C}(33)-\mathrm{C}(37)-\mathrm{U}(2)$ & $74.8(5)$ \\
\hline$C(25)-C(26)-C(30)$ & $118.9(8)$ & $\mathrm{C}(36)-\mathrm{C}(37)-\mathrm{U}(2)$ & $73.7(6)$ \\
\hline$C(21)-C(26)-C(30)$ & $122.3(8)$ & $\mathrm{C}(42)-\mathrm{C}(37)-\mathrm{U}(2)$ & $127.8(7)$ \\
\hline$C(22)-C(27)-C(29)$ & $114.5(7)$ & $C(44)-C(43)-C(47)$ & $104.9(8)$ \\
\hline $\mathrm{C}(22)-\mathrm{C}(27)-\mathrm{C}(28)$ & $111.4(8)$ & $\mathrm{C}(44)-\mathrm{C}(43)-\mathrm{C}(48)$ & $125.7(10)$ \\
\hline$C(29)-C(27)-C(28)$ & $108.6(7)$ & $\mathrm{C}(47)-\mathrm{C}(43)-\mathrm{C}(48)$ & $128.1(10)$ \\
\hline$C(26)-C(30)-C(31)$ & $110.7(7)$ & $\mathrm{C}(44)-\mathrm{C}(43)-\mathrm{U}(2)$ & $74.5(5)$ \\
\hline$C(26)-C(30)-C(32)$ & $113.7(8)$ & $\mathrm{C}(47)-\mathrm{C}(43)-\mathrm{U}(2)$ & $75.2(5)$ \\
\hline$C(31)-C(30)-C(32)$ & $109.5(8)$ & $\mathrm{C}(48)-\mathrm{C}(43)-\mathrm{U}(2)$ & $125.5(6)$ \\
\hline $\mathrm{C}(37)-\mathrm{C}(33)-\mathrm{C}(34)$ & $107.7(8)$ & $C(45)-C(44)-C(43)$ & $109.1(8)$ \\
\hline $\mathrm{C}(37)-\mathrm{C}(33)-\mathrm{C}(38)$ & $125.3(9)$ & $\mathrm{C}(45)-\mathrm{C}(44)-\mathrm{C}(49)$ & $126.5(9)$ \\
\hline $\mathrm{C}(34)-\mathrm{C}(33)-\mathrm{C}(38)$ & $126.8(9)$ & $C(43)-C(44)-C(49)$ & $123.8(10)$ \\
\hline $\mathrm{C}(37)-\mathrm{C}(33)-\mathrm{U}(2)$ & $75.6(5)$ & $\mathrm{C}(45)-\mathrm{C}(44)-\mathrm{U}(2)$ & $76.9(5)$ \\
\hline $\mathrm{C}(34)-\mathrm{C}(33)-\mathrm{U}(2)$ & $75.0(5)$ & $\mathrm{C}(43)-\mathrm{C}(44)-\mathrm{U}(2)$ & $75.1(5)$ \\
\hline $\mathrm{C}(38)-\mathrm{C}(33)-\mathrm{U}(2)$ & $119.6(6)$ & $\mathrm{C}(49)-\mathrm{C}(44)-\mathrm{U}(2)$ & $121.5(6)$ \\
\hline$C(33)-C(34)-C(35)$ & $108.1(8)$ & $C(46)-C(45)-C(44)$ & $107.4(8)$ \\
\hline $\mathrm{C}(33)-\mathrm{C}(34)-\mathrm{C}(39)$ & $126.2(9)$ & $\mathrm{C}(46)-\mathrm{C}(45)-\mathrm{C}(50)$ & $126.0(9)$ \\
\hline$C(35)-C(34)-C(39)$ & $124.8(9)$ & $C(44)-C(45)-C(50)$ & $126.3(9)$ \\
\hline C(33)-C(34)-U(2) & $75.2(5)$ & $\mathrm{C}(46)-\mathrm{C}(45)-\mathrm{U}(2)$ & $75.2(5)$ \\
\hline $\mathrm{C}(35)-\mathrm{C}(34)-\mathrm{U}(2)$ & $73.8(5)$ & $\mathrm{C}(44)-\mathrm{C}(45)-\mathrm{U}(2)$ & $73.4(5)$ \\
\hline C(39)-C(34)-U(2) & $125.4(6)$ & $\mathrm{C}(50)-\mathrm{C}(45)-\mathrm{U}(2)$ & $121.1(6)$ \\
\hline$C(36)-C(35)-C(34)$ & 107.2(9) & $\mathrm{C}(45)-\mathrm{C}(46)-\mathrm{C}(47)$ & $110.5(8)$ \\
\hline$C(36)-C(35)-C(40)$ & $129.0(10)$ & $C(45)-C(46)-C(51)$ & $122.3(9)$ \\
\hline$C(34)-C(35)-C(40)$ & $122.9(9)$ & $\mathrm{C}(47)-\mathrm{C}(46)-\mathrm{C}(51)$ & $125.9(9)$ \\
\hline $\mathrm{C}(36)-\mathrm{C}(35)-\mathrm{U}(2)$ & $75.1(6)$ & $\mathrm{C}(45)-\mathrm{C}(46)-\mathrm{U}(2)$ & $75.9(5)$ \\
\hline $\mathrm{C}(34)-\mathrm{C}(35)-\mathrm{U}(2)$ & $75.8(5)$ & $\mathrm{C}(47)-\mathrm{C}(46)-\mathrm{U}(2)$ & $75.0(5)$ \\
\hline $\mathrm{C}(51)-\mathrm{C}(46)-\mathrm{U}(2)$ & $126.7(6)$ & $\mathrm{C}(54)-\mathrm{C}(55)-\mathrm{C}(56)$ & $122.5(9)$ \\
\hline
\end{tabular}




$\begin{array}{lrll}\mathrm{C}(46)-\mathrm{C}(47)-\mathrm{C}(43) & 108.1(8) & \mathrm{C}(55)-\mathrm{C}(56)-\mathrm{C}(57) & 119.2(9) \\ \mathrm{C}(46)-\mathrm{C}(47)-\mathrm{C}(52) & 124.7(9) & \mathrm{C}(56)-\mathrm{C}(57)-\mathrm{C}(58) & 121.5(8) \\ \mathrm{C}(43)-\mathrm{C}(47)-\mathrm{C}(52) & 127.1(9) & \mathrm{C}(53)-\mathrm{C}(58)-\mathrm{C}(57) & 118.5(8) \\ \mathrm{C}(46)-\mathrm{C}(47)-\mathrm{U}(2) & 76.0(5) & \mathrm{C}(53)-\mathrm{C}(58)-\mathrm{C}(62) & 121.6(8) \\ \mathrm{C}(43)-\mathrm{C}(47)-\mathrm{U}(2) & 74.0(5) & \mathrm{C}(57)-\mathrm{C}(58)-\mathrm{C}(62) & 119.9(8) \\ \mathrm{C}(52)-\mathrm{C}(47)-\mathrm{U}(2) & 118.9(6) & \mathrm{C}(54)-\mathrm{C}(59)-\mathrm{C}(60) & 114.1(8) \\ \mathrm{C}(58)-\mathrm{C}(53)-\mathrm{N}(2) & 118.9(8) & \mathrm{C}(54)-\mathrm{C}(59)-\mathrm{C}(61) & 108.8(7) \\ \mathrm{C}(58)-\mathrm{C}(53)-\mathrm{C}(54) & 120.3(8) & \mathrm{C}(60)-\mathrm{C}(59)-\mathrm{C}(61) & 109.4(8) \\ \mathrm{N}(2)-\mathrm{C}(53)-\mathrm{C}(54) & 120.8(7) & \mathrm{C}(58)-\mathrm{C}(62)-\mathrm{C}(64) & 116.4(7) \\ \mathrm{C}(55)-\mathrm{C}(54)-\mathrm{C}(53) & 117.8(8) & \mathrm{C}(58)-\mathrm{C}(62)-\mathrm{C}(63) & 109.6(7) \\ \mathrm{C}(55)-\mathrm{C}(54)-\mathrm{C}(59) & 120.3(8) & \mathrm{C}(64)-\mathrm{C}(62)-\mathrm{C}(63) & 110.3(7) \\ \mathrm{C}(53)-\mathrm{C}(54)-\mathrm{C}(59) & 121.8(8) & & \end{array}$


Figure S3. Molecular structure of complex 5 with thermal ellipsoids at the 50\% probability level. Selected bond distances $(\AA)$ and angles $\left({ }^{\circ}\right)$ for 5: $\left.\mathrm{U}(1)-\mathrm{O}(1) 2.378(4)\right)$, U(1)-N(1) 1.957(5), N(1)-C(22) 1.416(8), U(1)- $\mathrm{C}_{5} \mathrm{Me}_{5 \text { (cent) }}$ 2.440(7), 2.435(7), N(1)-U(1)-O(1) 109.16(19), C(22)-N(1)-U(1) 168.3(5), $\mathrm{C}_{5} \mathrm{Me}_{5(\text { cent) }}-\mathrm{U}(1)-\mathrm{C}_{5} \mathrm{Me}_{5 \text { (cent) }} 135.9(2)$.

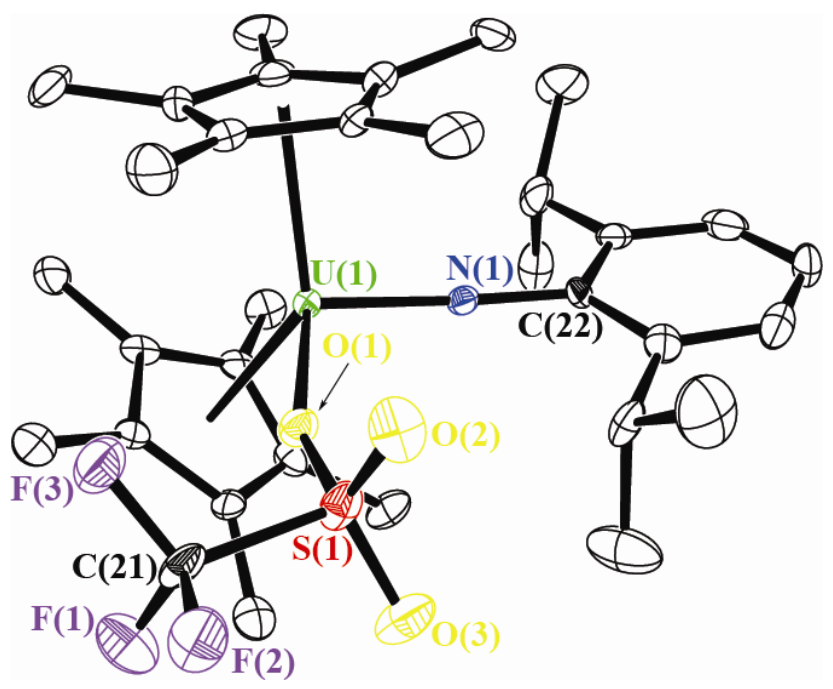


Table S5. Crystal Data and Structure Refinement for $\left(\mathrm{C}_{5} \mathrm{Me}_{5}\right)_{2} \mathrm{U}\left(=\mathrm{N}-2,6-{ }^{i} \mathrm{Pr}_{2}-\mathrm{C}_{6} \mathrm{H}_{3}\right)\left(\mathrm{OSO}_{2} \mathrm{CF}_{3}\right)($ 5).

Empirical formula

Formula weight

Temperature

Wavelength

Crystal system

Space group

Unit cell dimensions

Volume

Z

Density (calculated)

Absorption coefficient

$\mathrm{F}(000)$

Crystal size

Theta range for data collection

Index ranges

Reflections collected

Independent reflections

Completeness to theta $=25.00^{\circ}$

Max. and min. transmission

Refinement method

Data / restraints / parameters

Goodness-of-fit on F2

Final $\mathrm{R}$ indices $[\mathrm{I}>2 \operatorname{sigma}(\mathrm{I})]$

$\mathrm{R}$ indices (all data)

Largest diff. peak and hole
$\mathrm{C}_{33} \mathrm{H}_{47} \mathrm{~F}_{3} \mathrm{NO}_{3} \mathrm{SU}$

$832.81 \mathrm{~g} / \mathrm{mol}$

120(1) K

$0.71073 \AA$

triclinic

Pī

$a=10.005(2) \AA$

$\alpha=76.628(2)^{\circ}$

$b=10.514(2) \AA$

$\beta=77.703(2)^{\circ}$

$c=17.370(4) \AA$

$\gamma=68.935(2)^{\circ}$

1641.7(6) $\AA^{3}$

2

$1.685 \mathrm{Mg} / \mathrm{m}^{3}$

$5.057 \mathrm{~mm}^{-1}$

822

$0.18 \times 0.08 \times 0.02 \mathrm{~mm}^{3}$

2.11 to $27.30^{\circ}$

$-12<=\mathrm{h}<=12,-13<=\mathrm{k}<=13,-22<=\mathrm{l}<=22$

17733

$7175[R(i n t)=0.0626]$

$99.3 \%$

0.9056 and 0.4630

Full-matrix least-squares on $\mathrm{F}^{2}$

7175 / 0 / 324

1.065

$R 1=0.0429, w R 2=0.0955$

$R 1=0.0594, w R 2=0.1110$

1.881 and -1.921 e. $\AA^{-3}$ 
Table S6. Bond Lengths $[\AA]$ and Angles $\left[^{\circ}\right]$ for $\left(\mathrm{C}_{5} \mathrm{Me}_{5}\right)_{2} \mathrm{U}\left(=\mathrm{N}-2,6-{ }^{i} \mathrm{Pr}_{2}-\mathrm{C}_{6} \mathrm{H}_{3}\right)\left(\mathrm{OSO}_{2} \mathrm{CF}_{3}\right)(5)$.

\begin{tabular}{|c|c|c|c|}
\hline $\mathrm{U}(1)-\mathrm{N}(1)$ & $1.957(5)$ & $C(13)-C(14)$ & $1.425(9)$ \\
\hline $\mathrm{U}(1)-\mathrm{O}(1)$ & $2.378(4)$ & $C(13)-C(18)$ & $1.503(10)$ \\
\hline $\mathrm{U}(1)-\mathrm{C}(13)$ & $2.663(6)$ & $C(14)-C(15)$ & $1.413(9)$ \\
\hline $\mathrm{U}(1)-\mathrm{C}(4)$ & $2.695(7)$ & $C(14)-C(19)$ & $1.492(9)$ \\
\hline $\mathrm{U}(1)-\mathrm{C}(14)$ & $2.699(6)$ & $C(15)-C(20)$ & $1.495(9)$ \\
\hline $\mathrm{U}(1)-\mathrm{C}(2)$ & $2.714(7)$ & $C(22)-C(23)$ & $1.417(9)$ \\
\hline $\mathrm{U}(1)-\mathrm{C}(3)$ & $2.716(7)$ & $C(22)-C(27)$ & $1.428(9)$ \\
\hline $\mathrm{U}(1)-\mathrm{C}(5)$ & $2.734(7)$ & $C(23)-C(24)$ & $1.392(10)$ \\
\hline $\mathrm{U}(1)-\mathrm{C}(12)$ & $2.736(6)$ & $C(23)-C(28)$ & $1.516(10)$ \\
\hline $\mathrm{U}(1)-\mathrm{C}(15)$ & $2.739(7)$ & $C(24)-C(25)$ & $1.365(11)$ \\
\hline $\mathrm{U}(1)-\mathrm{C}(1)$ & $2.754(7)$ & $C(25)-C(26)$ & $1.390(10)$ \\
\hline $\mathrm{U}(1)-\mathrm{C}(11)$ & $2.753(6)$ & $C(26)-C(27)$ & $1.371(10)$ \\
\hline $\mathrm{S}(1)-\mathrm{O}(3)$ & $1.420(5)$ & $\mathrm{C}(27)-\mathrm{C}(31)$ & $1.510(10)$ \\
\hline $\mathrm{S}(1)-\mathrm{O}(2)$ & $1.422(5)$ & $\mathrm{C}(28)-\mathrm{C}(30)$ & $1.528(11)$ \\
\hline $\mathrm{S}(1)-\mathrm{O}(1)$ & $1.485(5)$ & $\mathrm{C}(28)-\mathrm{C}(29)$ & $1.542(10)$ \\
\hline$S(1)-C(21)$ & $1.838(7)$ & $\mathrm{C}(31)-\mathrm{C}(32)$ & $1.503(11)$ \\
\hline $\mathrm{F}(1)-\mathrm{C}(21)$ & $1.330(9)$ & $\mathrm{C}(31)-\mathrm{C}(33)$ & $1.519(11)$ \\
\hline $\mathrm{F}(2)-\mathrm{C}(21)$ & $1.323(8)$ & & \\
\hline $\mathrm{F}(3)-\mathrm{C}(21)$ & $1.323(8)$ & $\mathrm{N}(1)-\mathrm{U}(1)-\mathrm{O}(1)$ & 109.16(19) \\
\hline $\mathrm{N}(1)-\mathrm{C}(22)$ & $1.416(8)$ & $\mathrm{N}(1)-\mathrm{U}(1)-\mathrm{C}(13)$ & $130.8(2)$ \\
\hline $\mathrm{C}(1)-\mathrm{C}(2)$ & $1.419(10)$ & $\mathrm{O}(1)-\mathrm{U}(1)-\mathrm{C}(13)$ & $82.11(18)$ \\
\hline$C(1)-C(5)$ & $1.430(10)$ & $\mathrm{N}(1)-\mathrm{U}(1)-\mathrm{C}(4)$ & $91.0(2)$ \\
\hline$C(1)-C(6)$ & $1.488(9)$ & $\mathrm{O}(1)-\mathrm{U}(1)-\mathrm{C}(4)$ & $83.48(18)$ \\
\hline $\mathrm{C}(2)-\mathrm{C}(3)$ & $1.433(10)$ & $\mathrm{C}(13)-\mathrm{U}(1)-\mathrm{C}(4)$ & $138.2(2)$ \\
\hline$C(2)-C(7)$ & $1.501(10)$ & $\mathrm{N}(1)-\mathrm{U}(1)-\mathrm{C}(14)$ & $103.6(2)$ \\
\hline $\mathrm{C}(3)-\mathrm{C}(4)$ & $1.407(9)$ & $\mathrm{O}(1)-\mathrm{U}(1)-\mathrm{C}(14)$ & $75.95(18)$ \\
\hline$C(3)-C(8)$ & $1.480(10)$ & $\mathrm{C}(13)-\mathrm{U}(1)-\mathrm{C}(14)$ & $30.80(19)$ \\
\hline$C(4)-C(5)$ & $1.416(9)$ & $\mathrm{C}(4)-\mathrm{U}(1)-\mathrm{C}(14)$ & $157.7(2)$ \\
\hline$C(4)-C(9)$ & $1.498(9)$ & $\mathrm{N}(1)-\mathrm{U}(1)-\mathrm{C}(2)$ & $95.3(2)$ \\
\hline$C(5)-C(10)$ & $1.497(10)$ & $\mathrm{O}(1)-\mathrm{U}(1)-\mathrm{C}(2)$ & $128.03(19)$ \\
\hline $\mathrm{C}(11)-\mathrm{C}(12)$ & $1.419(10)$ & $\mathrm{C}(13)-\mathrm{U}(1)-\mathrm{C}(2)$ & $115.3(2)$ \\
\hline$C(11)-C(15)$ & $1.422(9)$ & $\mathrm{C}(4)-\mathrm{U}(1)-\mathrm{C}(2)$ & $50.1(2)$ \\
\hline$C(11)-C(16)$ & $1.510(9)$ & $\mathrm{C}(14)-\mathrm{U}(1)-\mathrm{C}(2)$ & $142.1(2)$ \\
\hline$C(12)-C(13)$ & $1.422(9)$ & $\mathrm{N}(1)-\mathrm{U}(1)-\mathrm{C}(3)$ & $75.8(2)$ \\
\hline $\mathrm{C}(12)-\mathrm{C}(17)$ & $1.503(9)$ & $\mathrm{O}(1)-\mathrm{U}(1)-\mathrm{C}(3)$ & 112.91(19) \\
\hline
\end{tabular}




\begin{tabular}{|c|c|c|c|}
\hline $\mathrm{C}(13)-\mathrm{U}(1)-\mathrm{C}(3)$ & $145.1(2)$ & $C(12)-U(1)-C(1)$ & $85.2(2)$ \\
\hline $\mathrm{C}(4)-\mathrm{U}(1)-\mathrm{C}(3)$ & $30.1(2)$ & $\mathrm{C}(15)-\mathrm{U}(1)-\mathrm{C}(1)$ & $133.7(2)$ \\
\hline $\mathrm{C}(14)-\mathrm{U}(1)-\mathrm{C}(3)$ & $170.9(2)$ & $\mathrm{N}(1)-\mathrm{U}(1)-\mathrm{C}(11)$ & $89.6(2)$ \\
\hline $\mathrm{C}(2)-\mathrm{U}(1)-\mathrm{C}(3)$ & $30.6(2)$ & $\mathrm{O}(1)-\mathrm{U}(1)-\mathrm{C}(11)$ & $125.67(18)$ \\
\hline $\mathrm{N}(1)-\mathrm{U}(1)-\mathrm{C}(5)$ & $120.9(2)$ & $\mathrm{C}(13)-\mathrm{U}(1)-\mathrm{C}(11)$ & $50.3(2)$ \\
\hline $\mathrm{O}(1)-\mathrm{U}(1)-\mathrm{C}(5)$ & $78.52(18)$ & $\mathrm{C}(4)-\mathrm{U}(1)-\mathrm{C}(11)$ & $148.62(19)$ \\
\hline $\mathrm{C}(13)-\mathrm{U}(1)-\mathrm{C}(5)$ & $108.1(2)$ & $\mathrm{C}(14)-\mathrm{U}(1)-\mathrm{C}(11)$ & $49.9(2)$ \\
\hline$C(4)-U(1)-C(5)$ & $30.2(2)$ & $\mathrm{C}(2)-\mathrm{U}(1)-\mathrm{C}(11)$ & $98.7(2)$ \\
\hline $\mathrm{C}(14)-\mathrm{U}(1)-\mathrm{C}(5)$ & $133.8(2)$ & $\mathrm{C}(3)-\mathrm{U}(1)-\mathrm{C}(11)$ & $121.1(2)$ \\
\hline $\mathrm{C}(2)-\mathrm{U}(1)-\mathrm{C}(5)$ & $49.8(2)$ & $\mathrm{C}(5)-\mathrm{U}(1)-\mathrm{C}(11)$ & $134.4(2)$ \\
\hline $\mathrm{C}(3)-\mathrm{U}(1)-\mathrm{C}(5)$ & $49.8(2)$ & $\mathrm{C}(12)-\mathrm{U}(1)-\mathrm{C}(11)$ & $30.0(2)$ \\
\hline $\mathrm{N}(1)-\mathrm{U}(1)-\mathrm{C}(12)$ & 119.4(2) & $\mathrm{C}(15)-\mathrm{U}(1)-\mathrm{C}(11)$ & $30.0(2)$ \\
\hline $\mathrm{O}(1)-\mathrm{U}(1)-\mathrm{C}(12)$ & $112.35(17)$ & $\mathrm{C}(1)-\mathrm{U}(1)-\mathrm{C}(11)$ & $105.4(2)$ \\
\hline $\mathrm{C}(13)-\mathrm{U}(1)-\mathrm{C}(12)$ & $30.51(19)$ & $\mathrm{O}(3)-\mathrm{S}(1)-\mathrm{O}(2)$ & 118.1(3) \\
\hline $\mathrm{C}(4)-\mathrm{U}(1)-\mathrm{C}(12)$ & $135.15(19)$ & $\mathrm{O}(3)-\mathrm{S}(1)-\mathrm{O}(1)$ & $113.1(3)$ \\
\hline $\mathrm{C}(14)-\mathrm{U}(1)-\mathrm{C}(12)$ & $49.98(19)$ & $\mathrm{O}(2)-\mathrm{S}(1)-\mathrm{O}(1)$ & $113.2(3)$ \\
\hline $\mathrm{C}(2)-\mathrm{U}(1)-\mathrm{C}(12)$ & $92.1(2)$ & $\mathrm{O}(3)-\mathrm{S}(1)-\mathrm{C}(21)$ & $103.8(3)$ \\
\hline$C(3)-U(1)-C(12)$ & $122.2(2)$ & $\mathrm{O}(2)-\mathrm{S}(1)-\mathrm{C}(21)$ & $104.4(3)$ \\
\hline$C(5)-U(1)-C(12)$ & $109.2(2)$ & $\mathrm{O}(1)-\mathrm{S}(1)-\mathrm{C}(21)$ & $101.9(3)$ \\
\hline $\mathrm{N}(1)-\mathrm{U}(1)-\mathrm{C}(15)$ & $80.6(2)$ & $\mathrm{S}(1)-\mathrm{O}(1)-\mathrm{U}(1)$ & $160.4(3)$ \\
\hline $\mathrm{O}(1)-\mathrm{U}(1)-\mathrm{C}(15)$ & $101.65(18)$ & $\mathrm{C}(22)-\mathrm{N}(1)-\mathrm{U}(1)$ & $168.3(5)$ \\
\hline $\mathrm{C}(13)-\mathrm{U}(1)-\mathrm{C}(15)$ & $50.3(2)$ & $C(2)-C(1)-C(5)$ & $107.2(6)$ \\
\hline $\mathrm{C}(4)-\mathrm{U}(1)-\mathrm{C}(15)$ & 171.2(2) & $C(2)-C(1)-C(6)$ & $127.8(7)$ \\
\hline$C(14)-U(1)-C(15)$ & $30.1(2)$ & $C(5)-C(1)-C(6)$ & $123.8(7)$ \\
\hline $\mathrm{C}(2)-\mathrm{U}(1)-\mathrm{C}(15)$ & $127.7(2)$ & $C(2)-C(1)-U(1)$ & 73.4(4) \\
\hline$C(3)-U(1)-C(15)$ & $142.9(2)$ & $C(5)-C(1)-U(1)$ & 74.1(4) \\
\hline$C(5)-U(1)-C(15)$ & $157.5(2)$ & $\mathrm{C}(6)-\mathrm{C}(1)-\mathrm{U}(1)$ & $127.5(5)$ \\
\hline$C(12)-U(1)-C(15)$ & $49.5(2)$ & $C(1)-C(2)-C(3)$ & $108.4(6)$ \\
\hline $\mathrm{N}(1)-\mathrm{U}(1)-\mathrm{C}(1)$ & $124.0(2)$ & $C(1)-C(2)-C(7)$ & $125.8(7)$ \\
\hline $\mathrm{O}(1)-\mathrm{U}(1)-\mathrm{C}(1)$ & $104.52(18)$ & $C(3)-C(2)-C(7)$ & $125.6(7)$ \\
\hline$C(13)-U(1)-C(1)$ & $96.7(2)$ & $C(1)-C(2)-U(1)$ & $76.5(4)$ \\
\hline$C(4)-U(1)-C(1)$ & 49.98(19) & $\mathrm{C}(3)-\mathrm{C}(2)-\mathrm{U}(1)$ & $74.8(4)$ \\
\hline $\mathrm{C}(14)-\mathrm{U}(1)-\mathrm{C}(1)$ & $127.5(2)$ & $C(7)-C(2)-U(1)$ & $119.6(5)$ \\
\hline $\mathrm{C}(2)-\mathrm{U}(1)-\mathrm{C}(1)$ & $30.1(2)$ & $C(4)-C(3)-C(2)$ & $107.4(6)$ \\
\hline $\mathrm{C}(3)-\mathrm{U}(1)-\mathrm{C}(1)$ & $50.02(19)$ & $\mathrm{C}(4)-\mathrm{C}(3)-\mathrm{C}(8)$ & $125.3(7)$ \\
\hline $\mathrm{C}(5)-\mathrm{U}(1)-\mathrm{C}(1)$ & $30.2(2)$ & $\mathrm{C}(2)-\mathrm{C}(3)-\mathrm{C}(8)$ & $126.5(7)$ \\
\hline
\end{tabular}




\begin{tabular}{|c|c|c|c|}
\hline $\mathrm{C}(4)-\mathrm{C}(3)-\mathrm{U}(1)$ & 74.1(4) & $\mathrm{C}(15)-\mathrm{C}(14)-\mathrm{U}(1)$ & $76.5(4)$ \\
\hline $\mathrm{C}(2)-\mathrm{C}(3)-\mathrm{U}(1)$ & $74.6(4)$ & $\mathrm{C}(13)-\mathrm{C}(14)-\mathrm{U}(1)$ & $73.2(4)$ \\
\hline $\mathrm{C}(8)-\mathrm{C}(3)-\mathrm{U}(1)$ & $124.8(5)$ & $\mathrm{C}(19)-\mathrm{C}(14)-\mathrm{U}(1)$ & $120.8(4)$ \\
\hline$C(3)-C(4)-C(5)$ & $108.8(6)$ & $C(14)-C(15)-C(11)$ & $108.4(6)$ \\
\hline$C(3)-C(4)-C(9)$ & $124.2(6)$ & $C(14)-C(15)-C(20)$ & $125.1(6)$ \\
\hline$C(5)-C(4)-C(9)$ & $126.7(7)$ & $\mathrm{C}(11)-\mathrm{C}(15)-\mathrm{C}(20)$ & $126.2(6)$ \\
\hline $\mathrm{C}(3)-\mathrm{C}(4)-\mathrm{U}(1)$ & $75.8(4)$ & $C(14)-C(15)-U(1)$ & $73.4(4)$ \\
\hline$C(5)-C(4)-U(1)$ & $76.4(4)$ & $\mathrm{C}(11)-\mathrm{C}(15)-\mathrm{U}(1)$ & $75.6(4)$ \\
\hline $\mathrm{C}(9)-\mathrm{C}(4)-\mathrm{U}(1)$ & $119.0(4)$ & $\mathrm{C}(20)-\mathrm{C}(15)-\mathrm{U}(1)$ & $122.3(5)$ \\
\hline$C(4)-C(5)-C(1)$ & $108.0(6)$ & $\mathrm{F}(3)-\mathrm{C}(21)-\mathrm{F}(2)$ & $107.9(6)$ \\
\hline $\mathrm{C}(4)-\mathrm{C}(5)-\mathrm{C}(10)$ & $125.9(7)$ & $\mathrm{F}(3)-\mathrm{C}(21)-\mathrm{F}(1)$ & $108.6(6)$ \\
\hline$C(1)-C(5)-C(10)$ & $125.9(7)$ & $\mathrm{F}(2)-\mathrm{C}(21)-\mathrm{F}(1)$ & $107.4(6)$ \\
\hline$C(4)-C(5)-U(1)$ & $73.4(4)$ & $\mathrm{F}(3)-\mathrm{C}(21)-\mathrm{S}(1)$ & $111.5(5)$ \\
\hline$C(1)-C(5)-U(1)$ & $75.7(4)$ & $\mathrm{F}(2)-\mathrm{C}(21)-\mathrm{S}(1)$ & $109.9(5)$ \\
\hline $\mathrm{C}(10)-\mathrm{C}(5)-\mathrm{U}(1)$ & $121.2(5)$ & $\mathrm{F}(1)-\mathrm{C}(21)-\mathrm{S}(1)$ & $111.4(5)$ \\
\hline $\mathrm{C}(12)-\mathrm{C}(11)-\mathrm{C}(15)$ & $107.6(6)$ & $\mathrm{N}(1)-\mathrm{C}(22)-\mathrm{C}(23)$ & $121.7(6)$ \\
\hline$C(12)-C(11)-C(16)$ & $124.9(6)$ & $\mathrm{N}(1)-\mathrm{C}(22)-\mathrm{C}(27)$ & $118.6(6)$ \\
\hline$C(15)-C(11)-C(16)$ & $126.5(6)$ & $C(23)-C(22)-C(27)$ & $119.7(6)$ \\
\hline $\mathrm{C}(12)-\mathrm{C}(11)-\mathrm{U}(1)$ & $74.3(4)$ & $\mathrm{C}(24)-\mathrm{C}(23)-\mathrm{C}(22)$ & $118.3(7)$ \\
\hline $\mathrm{C}(15)-\mathrm{C}(11)-\mathrm{U}(1)$ & $74.4(4)$ & $C(24)-C(23)-C(28)$ & $119.5(6)$ \\
\hline $\mathrm{C}(16)-\mathrm{C}(11)-\mathrm{U}(1)$ & $126.0(4)$ & $\mathrm{C}(22)-\mathrm{C}(23)-\mathrm{C}(28)$ & $122.1(6)$ \\
\hline $\mathrm{C}(11)-\mathrm{C}(12)-\mathrm{C}(13)$ & $108.4(6)$ & $\mathrm{C}(25)-\mathrm{C}(24)-\mathrm{C}(23)$ & $122.4(7)$ \\
\hline $\mathrm{C}(11)-\mathrm{C}(12)-\mathrm{C}(17)$ & $123.1(6)$ & $\mathrm{C}(24)-\mathrm{C}(25)-\mathrm{C}(26)$ & $118.8(7)$ \\
\hline $\mathrm{C}(13)-\mathrm{C}(12)-\mathrm{C}(17)$ & $127.5(6)$ & $\mathrm{C}(27)-\mathrm{C}(26)-\mathrm{C}(25)$ & $122.5(7)$ \\
\hline $\mathrm{C}(11)-\mathrm{C}(12)-\mathrm{U}(1)$ & $75.7(4)$ & $\mathrm{C}(26)-\mathrm{C}(27)-\mathrm{C}(22)$ & $118.3(7)$ \\
\hline $\mathrm{C}(13)-\mathrm{C}(12)-\mathrm{U}(1)$ & $71.9(3)$ & $\mathrm{C}(26)-\mathrm{C}(27)-\mathrm{C}(31)$ & $121.1(6)$ \\
\hline $\mathrm{C}(17)-\mathrm{C}(12)-\mathrm{U}(1)$ & $127.6(4)$ & $\mathrm{C}(22)-\mathrm{C}(27)-\mathrm{C}(31)$ & $120.5(6)$ \\
\hline$C(14)-C(13)-C(12)$ & $107.5(6)$ & $\mathrm{C}(23)-\mathrm{C}(28)-\mathrm{C}(30)$ & $111.4(6)$ \\
\hline$C(14)-C(13)-C(18)$ & $127.5(6)$ & $\mathrm{C}(23)-\mathrm{C}(28)-\mathrm{C}(29)$ & $112.7(6)$ \\
\hline $\mathrm{C}(12)-\mathrm{C}(13)-\mathrm{C}(18)$ & $124.9(6)$ & $\mathrm{C}(30)-\mathrm{C}(28)-\mathrm{C}(29)$ & $108.3(6)$ \\
\hline $\mathrm{C}(14)-\mathrm{C}(13)-\mathrm{U}(1)$ & $76.0(4)$ & $C(32)-C(31)-C(27)$ & $110.2(6)$ \\
\hline $\mathrm{C}(12)-\mathrm{C}(13)-\mathrm{U}(1)$ & $77.6(4)$ & $\mathrm{C}(32)-\mathrm{C}(31)-\mathrm{C}(33)$ & $110.9(7)$ \\
\hline $\mathrm{C}(18)-\mathrm{C}(13)-\mathrm{U}(1)$ & $112.2(5)$ & $\mathrm{C}(27)-\mathrm{C}(31)-\mathrm{C}(33)$ & $114.5(6)$ \\
\hline$C(15)-C(14)-C(13)$ & $108.1(6)$ & & \\
\hline$C(15)-C(14)-C(19)$ & $123.9(6)$ & & \\
\hline$C(13)-C(14)-C(19)$ & $127.8(6)$ & & \\
\hline
\end{tabular}


Figure S4. Molecular structure of complex 6 with thermal ellipsoids at the $50 \%$ probability level.

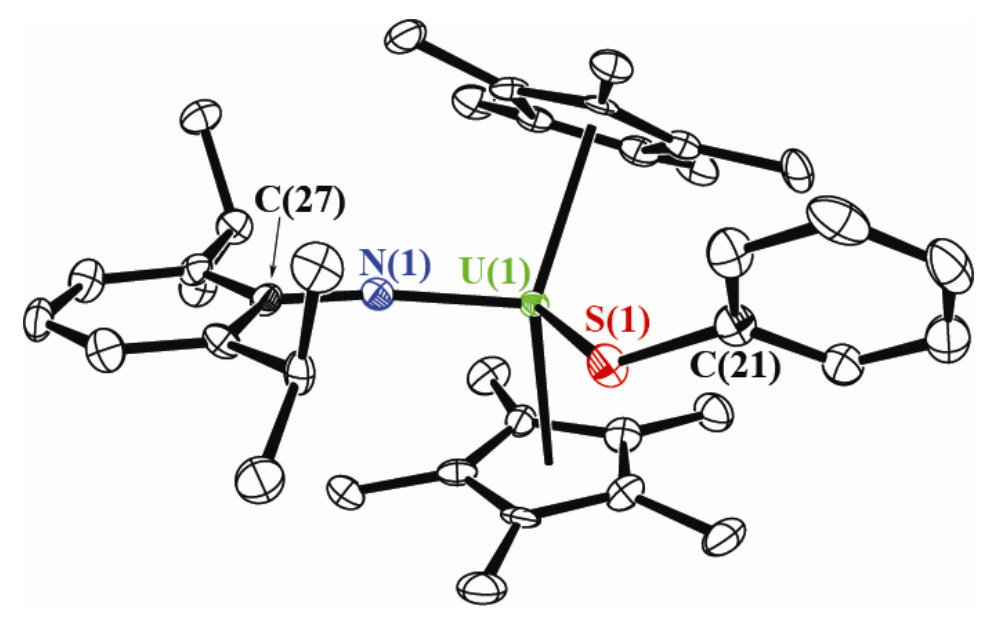


Table S7. Crystal Data and Structure Refinement for $\left(\mathrm{C}_{5} \mathrm{Me}_{5}\right)_{2} \mathrm{U}\left(=\mathrm{N}-2,6-{ }^{i} \mathrm{Pr}_{2}-\mathrm{C}_{6} \mathrm{H}_{3}\right)(\mathrm{SPh})(\mathbf{6})$.

Empirical formula

Formula weight

Temperature

Wavelength

Crystal system

Space group

Unit cell dimensions

Volume

Z

Density (calculated)

Absorption coefficient

$\mathrm{F}(000)$

Crystal size

Theta range for data collection

Index ranges

Reflections collected

Independent reflections

Completeness to theta $=25.00^{\circ}$

Max. and min. transmission

Refinement method

Data / restraints / parameters

Goodness-of-fit on F2

Final $\mathrm{R}$ indices $[\mathrm{I}>2 \operatorname{sigma}(\mathrm{I})]$

$\mathrm{R}$ indices (all data)

Largest diff. peak and hole
$\mathrm{C}_{38} \mathrm{H}_{52} \mathrm{NSU}$

$792.90 \mathrm{~g} / \mathrm{mol}$

120(1) K

$0.71073 \AA$

Monoclinic

$\mathrm{P} 2{ }_{1} / \mathrm{n}$

$a=12.0083(14) \AA$

$\alpha=90^{\circ}$

$b=18.205(2) \AA$

$\beta=99.049(1)^{\circ}$

$c=15.6133(18) \AA$

$\gamma=90^{\circ}$

3370.7(7) $\AA^{3}$

4

$1.562 \mathrm{Mg} / \mathrm{m}^{3}$

$4.903 \mathrm{~mm}^{-1}$

1580

$0.20 \times 0.14 \times 0.06 \mathrm{~mm}^{3}$

2.00 to $28.27^{\circ}$.

$-15<=\mathrm{h}<=15,-23<=\mathrm{k}<=23,-20<=1<=20$

36462

$7709[R($ int $)=0.0570]$

$99.6 \%$

0.7574 and 0.4405

Full-matrix least-squares on $\mathrm{F}^{2}$

7709 / 0 / 384

1.305

$R 1=0.0316, w R 2=0.0830$

$R 1=0.0458, w R 2=0.1038$

1.455 and -1.743 e. $\AA^{-3}$ 
Table S8. Bond Lengths $[\AA]$ and Angles $\left[^{\circ}\right]$ for $\left(\mathrm{C}_{5} \mathrm{Me}_{5}\right)_{2} \mathrm{U}\left(=\mathrm{N}-2,6-{ }^{i} \mathrm{Pr}_{2}-\mathrm{C}_{6} \mathrm{H}_{3}\right)(\mathrm{SPh})(6)$

\begin{tabular}{|c|c|c|c|}
\hline $\mathrm{U}(1)-\mathrm{N}(1)$ & $1.976(4)$ & $C(21)-C(22)$ & $1.386(8)$ \\
\hline $\mathrm{U}(1)-\mathrm{S}(1)$ & $2.7230(13)$ & $C(21)-C(26)$ & $1.397(8)$ \\
\hline $\mathrm{U}(1)-\mathrm{C}(14)$ & $2.724(5)$ & $C(22)-C(23)$ & $1.392(8)$ \\
\hline $\mathrm{U}(1)-\mathrm{C}(1)$ & $2.726(4)$ & $C(23)-C(24)$ & $1.376(9)$ \\
\hline $\mathrm{U}(1)-\mathrm{C}(4)$ & $2.732(4)$ & $C(24)-C(25)$ & $1.374(9)$ \\
\hline $\mathrm{U}(1)-\mathrm{C}(5)$ & $2.731(5)$ & $C(25)-C(26)$ & $1.399(8)$ \\
\hline $\mathrm{U}(1)-\mathrm{C}(13)$ & $2.738(5)$ & $C(27)-C(28)$ & $1.419(7)$ \\
\hline $\mathrm{U}(1)-\mathrm{C}(3)$ & $2.743(5)$ & $C(27)-C(32)$ & $1.425(7)$ \\
\hline $\mathrm{U}(1)-\mathrm{C}(12)$ & $2.743(5)$ & $C(28)-C(29)$ & $1.391(7)$ \\
\hline U(1)-C(15) & $2.747(5)$ & $C(28)-C(33)$ & $1.526(7)$ \\
\hline $\mathrm{U}(1)-\mathrm{C}(2)$ & $2.755(5)$ & $C(29)-C(30)$ & $1.385(7)$ \\
\hline $\mathrm{U}(1)-\mathrm{C}(11)$ & $2.772(5)$ & $\mathrm{C}(30)-\mathrm{C}(31)$ & $1.382(7)$ \\
\hline $\mathrm{S}(1)-\mathrm{C}(21)$ & $1.775(5)$ & $\mathrm{C}(31)-\mathrm{C}(32)$ & $1.393(7)$ \\
\hline N(1)-C(27) & $1.398(6)$ & $C(32)-C(36)$ & $1.519(7)$ \\
\hline$C(1)-C(5)$ & $1.409(6)$ & $\mathrm{C}(33)-\mathrm{C}(34)$ & $1.532(7)$ \\
\hline$C(1)-C(2)$ & $1.421(7)$ & $C(33)-C(35)$ & $1.533(7)$ \\
\hline$C(1)-C(6)$ & $1.506(7)$ & $C(36)-C(38)$ & $1.520(7)$ \\
\hline$C(2)-C(3)$ & $1.429(7)$ & $C(36)-C(37)$ & $1.531(7)$ \\
\hline$C(2)-C(7)$ & $1.503(7)$ & & \\
\hline$C(3)-C(4)$ & $1.420(7)$ & $\mathrm{N}(1)-\mathrm{U}(1)-\mathrm{S}(1)$ & $103.35(12)$ \\
\hline$C(3)-C(8)$ & $1.496(7)$ & $\mathrm{N}(1)-\mathrm{U}(1)-\mathrm{C}(14)$ & $90.72(17)$ \\
\hline$C(4)-C(5)$ & $1.411(7)$ & $\mathrm{S}(1)-\mathrm{U}(1)-\mathrm{C}(14)$ & $80.60(12)$ \\
\hline$C(4)-C(9)$ & $1.496(7)$ & $\mathrm{N}(1)-\mathrm{U}(1)-\mathrm{C}(1)$ & $89.91(16)$ \\
\hline$C(5)-C(10)$ & $1.514(7)$ & $\mathrm{S}(1)-\mathrm{U}(1)-\mathrm{C}(1)$ & $131.03(11)$ \\
\hline$C(11)-C(15)$ & $1.400(8)$ & $\mathrm{C}(14)-\mathrm{U}(1)-\mathrm{C}(1)$ & $147.09(15)$ \\
\hline $\mathrm{C}(11)-\mathrm{C}(12)$ & $1.428(7)$ & $\mathrm{N}(1)-\mathrm{U}(1)-\mathrm{C}(4)$ & $101.78(16)$ \\
\hline$C(11)-C(16)$ & $1.512(8)$ & $\mathrm{S}(1)-\mathrm{U}(1)-\mathrm{C}(4)$ & $81.40(11)$ \\
\hline$C(12)-C(13)$ & $1.415(8)$ & $\mathrm{C}(14)-\mathrm{U}(1)-\mathrm{C}(4)$ & $160.04(16)$ \\
\hline $\mathrm{C}(12)-\mathrm{C}(17)$ & $1.495(8)$ & $\mathrm{C}(1)-\mathrm{U}(1)-\mathrm{C}(4)$ & $49.65(14)$ \\
\hline$C(13)-C(14)$ & $1.435(8)$ & $\mathrm{N}(1)-\mathrm{U}(1)-\mathrm{C}(5)$ & $79.66(16)$ \\
\hline $\mathrm{C}(13)-\mathrm{C}(18)$ & $1.500(7)$ & $\mathrm{S}(1)-\mathrm{U}(1)-\mathrm{C}(5)$ & $105.97(10)$ \\
\hline$C(14)-C(15)$ & $1.408(8)$ & $\mathrm{C}(14)-\mathrm{U}(1)-\mathrm{C}(5)$ & $169.33(16)$ \\
\hline$C(14)-C(19)$ & $1.497(8)$ & $\mathrm{C}(1)-\mathrm{U}(1)-\mathrm{C}(5)$ & $29.91(14)$ \\
\hline$C(15)-C(20)$ & $1.500(7)$ & $\mathrm{C}(4)-\mathrm{U}(1)-\mathrm{C}(5)$ & $29.94(14)$ \\
\hline $\mathrm{N}(1)-\mathrm{U}(1)-\mathrm{C}(13)$ & $75.02(16)$ & $\mathrm{C}(13)-\mathrm{U}(1)-\mathrm{C}(2)$ & $122.65(15)$ \\
\hline
\end{tabular}




\begin{tabular}{|c|c|c|c|}
\hline$S(1)-U(1)-C(13)$ & $109.43(12)$ & $C(3)-U(1)-C(2)$ & $30.14(14)$ \\
\hline $\mathrm{C}(14)-\mathrm{U}(1)-\mathrm{C}(13)$ & $30.45(16)$ & $\mathrm{C}(12)-\mathrm{U}(1)-\mathrm{C}(2)$ & $93.15(16)$ \\
\hline $\mathrm{C}(1)-\mathrm{U}(1)-\mathrm{C}(13)$ & $119.54(15)$ & $C(15)-U(1)-C(2)$ & $108.99(16)$ \\
\hline $\mathrm{C}(4)-\mathrm{U}(1)-\mathrm{C}(13)$ & $169.09(15)$ & $\mathrm{N}(1)-\mathrm{U}(1)-\mathrm{C}(11)$ & $122.21(16)$ \\
\hline $\mathrm{C}(5)-\mathrm{U}(1)-\mathrm{C}(13)$ & $140.17(15)$ & $\mathrm{S}(1)-\mathrm{U}(1)-\mathrm{C}(11)$ & $107.20(13)$ \\
\hline $\mathrm{N}(1)-\mathrm{U}(1)-\mathrm{C}(3)$ & $128.84(16)$ & $C(14)-U(1)-C(11)$ & $49.14(16)$ \\
\hline$S(1)-U(1)-C(3)$ & $88.19(11)$ & $\mathrm{C}(1)-\mathrm{U}(1)-\mathrm{C}(11)$ & $104.39(16)$ \\
\hline $\mathrm{C}(14)-\mathrm{U}(1)-\mathrm{C}(3)$ & $140.44(16)$ & $\mathrm{C}(4)-\mathrm{U}(1)-\mathrm{C}(11)$ & $130.20(16)$ \\
\hline$C(1)-U(1)-C(3)$ & $49.74(15)$ & $\mathrm{C}(5)-\mathrm{U}(1)-\mathrm{C}(11)$ & $133.41(16)$ \\
\hline $\mathrm{C}(4)-\mathrm{U}(1)-\mathrm{C}(3)$ & $30.07(14)$ & $\mathrm{C}(13)-\mathrm{U}(1)-\mathrm{C}(11)$ & $48.97(16)$ \\
\hline$C(5)-U(1)-C(3)$ & $49.45(15)$ & $\mathrm{C}(3)-\mathrm{U}(1)-\mathrm{C}(11)$ & $100.18(16)$ \\
\hline $\mathrm{C}(13)-\mathrm{U}(1)-\mathrm{C}(3)$ & $147.29(15)$ & $\mathrm{C}(12)-\mathrm{U}(1)-\mathrm{C}(11)$ & $30.01(15)$ \\
\hline $\mathrm{N}(1)-\mathrm{U}(1)-\mathrm{C}(12)$ & $93.67(16)$ & $C(15)-U(1)-C(11)$ & $29.39(17)$ \\
\hline$S(1)-U(1)-C(12)$ & $128.01(12)$ & $C(2)-U(1)-C(11)$ & $86.12(16)$ \\
\hline $\mathrm{C}(14)-\mathrm{U}(1)-\mathrm{C}(12)$ & $50.03(16)$ & $\mathrm{C}(21)-\mathrm{S}(1)-\mathrm{U}(1)$ & $131.08(17)$ \\
\hline $\mathrm{C}(1)-\mathrm{U}(1)-\mathrm{C}(12)$ & $97.10(15)$ & $\mathrm{C}(27)-\mathrm{N}(1)-\mathrm{U}(1)$ & 171.6(3) \\
\hline $\mathrm{C}(4)-\mathrm{U}(1)-\mathrm{C}(12)$ & $142.53(15)$ & $C(5)-C(1)-C(2)$ & $108.0(4)$ \\
\hline $\mathrm{C}(5)-\mathrm{U}(1)-\mathrm{C}(12)$ & $125.43(15)$ & $C(5)-C(1)-C(6)$ & $127.3(5)$ \\
\hline $\mathrm{C}(13)-\mathrm{U}(1)-\mathrm{C}(12)$ & $29.91(16)$ & $C(2)-C(1)-C(6)$ & $123.7(4)$ \\
\hline $\mathrm{C}(3)-\mathrm{U}(1)-\mathrm{C}(12)$ & $117.97(15)$ & $C(5)-C(1)-U(1)$ & $75.2(3)$ \\
\hline $\mathrm{N}(1)-\mathrm{U}(1)-\mathrm{C}(15)$ & $120.04(17)$ & $C(2)-C(1)-U(1)$ & $76.1(3)$ \\
\hline$S(1)-U(1)-C(15)$ & $80.13(12)$ & $C(6)-C(1)-U(1)$ & $123.7(3)$ \\
\hline$C(14)-U(1)-C(15)$ & $29.82(16)$ & $C(1)-C(2)-C(3)$ & $107.6(4)$ \\
\hline$C(1)-U(1)-C(15)$ & $132.64(15)$ & $C(1)-C(2)-C(7)$ & $124.7(5)$ \\
\hline $\mathrm{C}(4)-\mathrm{U}(1)-\mathrm{C}(15)$ & $137.08(16)$ & $C(3)-C(2)-C(7)$ & $126.7(5)$ \\
\hline $\mathrm{C}(5)-\mathrm{U}(1)-\mathrm{C}(15)$ & $158.07(16)$ & $C(1)-C(2)-U(1)$ & $73.9(3)$ \\
\hline $\mathrm{C}(13)-\mathrm{U}(1)-\mathrm{C}(15)$ & $49.24(16)$ & $\mathrm{C}(3)-\mathrm{C}(2)-\mathrm{U}(1)$ & $74.4(3)$ \\
\hline $\mathrm{C}(3)-\mathrm{U}(1)-\mathrm{C}(15)$ & $110.97(16)$ & $C(7)-C(2)-U(1)$ & $126.7(3)$ \\
\hline $\mathrm{C}(12)-\mathrm{U}(1)-\mathrm{C}(15)$ & $49.43(15)$ & $C(4)-C(3)-C(2)$ & $107.7(4)$ \\
\hline $\mathrm{N}(1)-\mathrm{U}(1)-\mathrm{C}(2)$ & $119.93(16)$ & $C(4)-C(3)-C(8)$ & $127.5(5)$ \\
\hline$S(1)-U(1)-C(2)$ & $117.89(11)$ & $\mathrm{C}(2)-\mathrm{C}(3)-\mathrm{C}(8)$ & $124.7(5)$ \\
\hline $\mathrm{C}(14)-\mathrm{U}(1)-\mathrm{C}(2)$ & $135.24(15)$ & $\mathrm{C}(4)-\mathrm{C}(3)-\mathrm{U}(1)$ & $74.5(3)$ \\
\hline $\mathrm{C}(1)-\mathrm{U}(1)-\mathrm{C}(2)$ & $30.04(15)$ & $C(2)-C(3)-U(1)$ & $75.4(3)$ \\
\hline$C(4)-U(1)-C(2)$ & $49.59(14)$ & $\mathrm{C}(8)-\mathrm{C}(3)-\mathrm{U}(1)$ & $118.3(3)$ \\
\hline$C(5)-U(1)-C(2)$ & $49.33(15)$ & $C(5)-C(4)-C(3)$ & $107.9(4)$ \\
\hline$C(5)-C(4)-C(9)$ & $123.9(4)$ & $\mathrm{C}(19)-\mathrm{C}(14)-\mathrm{U}(1)$ & $118.2(3)$ \\
\hline
\end{tabular}




\begin{tabular}{|c|c|c|c|}
\hline $\mathrm{C}(3)-\mathrm{C}(4)-\mathrm{C}(9)$ & $127.5(5)$ & $\mathrm{C}(11)-\mathrm{C}(15)-\mathrm{C}(14)$ & $109.0(5)$ \\
\hline$C(5)-C(4)-U(1)$ & $75.0(3)$ & $C(11)-C(15)-C(20)$ & $125.1(5)$ \\
\hline $\mathrm{C}(3)-\mathrm{C}(4)-\mathrm{U}(1)$ & $75.4(3)$ & $C(14)-C(15)-C(20)$ & $125.3(5)$ \\
\hline $\mathrm{C}(9)-\mathrm{C}(4)-\mathrm{U}(1)$ & $123.2(3)$ & $\mathrm{C}(11)-\mathrm{C}(15)-\mathrm{U}(1)$ & $76.3(3)$ \\
\hline$C(1)-C(5)-C(4)$ & $108.7(4)$ & $\mathrm{C}(14)-\mathrm{C}(15)-\mathrm{U}(1)$ & $74.2(3)$ \\
\hline $\mathrm{C}(1)-\mathrm{C}(5)-\mathrm{C}(10)$ & $127.0(4)$ & $\mathrm{C}(20)-\mathrm{C}(15)-\mathrm{U}(1)$ & $122.7(4)$ \\
\hline$C(4)-C(5)-C(10)$ & $124.1(4)$ & $C(22)-C(21)-C(26)$ & $118.6(5)$ \\
\hline $\mathrm{C}(1)-\mathrm{C}(5)-\mathrm{U}(1)$ & $74.8(3)$ & $C(22)-C(21)-S(1)$ & $121.5(4)$ \\
\hline $\mathrm{C}(4)-\mathrm{C}(5)-\mathrm{U}(1)$ & $75.0(3)$ & $C(26)-C(21)-S(1)$ & $119.9(4)$ \\
\hline $\mathrm{C}(10)-\mathrm{C}(5)-\mathrm{U}(1)$ & $120.6(3)$ & $\mathrm{C}(21)-\mathrm{C}(22)-\mathrm{C}(23)$ & $120.3(6)$ \\
\hline $\mathrm{C}(15)-\mathrm{C}(11)-\mathrm{C}(12)$ & $108.5(5)$ & $\mathrm{C}(24)-\mathrm{C}(23)-\mathrm{C}(22)$ & $121.1(6)$ \\
\hline$C(15)-C(11)-C(16)$ & $124.4(5)$ & $\mathrm{C}(25)-\mathrm{C}(24)-\mathrm{C}(23)$ & $119.2(6)$ \\
\hline$C(12)-C(11)-C(16)$ & $126.3(5)$ & $C(24)-C(25)-C(26)$ & $120.5(6)$ \\
\hline $\mathrm{C}(15)-\mathrm{C}(11)-\mathrm{U}(1)$ & $74.3(3)$ & $\mathrm{C}(21)-\mathrm{C}(26)-\mathrm{C}(25)$ & $120.3(5)$ \\
\hline $\mathrm{C}(12)-\mathrm{C}(11)-\mathrm{U}(1)$ & $73.9(3)$ & $\mathrm{N}(1)-\mathrm{C}(27)-\mathrm{C}(28)$ & $122.3(4)$ \\
\hline $\mathrm{C}(16)-\mathrm{C}(11)-\mathrm{U}(1)$ & $125.9(4)$ & $\mathrm{N}(1)-\mathrm{C}(27)-\mathrm{C}(32)$ & $119.0(4)$ \\
\hline $\mathrm{C}(13)-\mathrm{C}(12)-\mathrm{C}(11)$ & $106.9(5)$ & $\mathrm{C}(28)-\mathrm{C}(27)-\mathrm{C}(32)$ & $118.7(4)$ \\
\hline$C(13)-C(12)-C(17)$ & $126.0(5)$ & $\mathrm{C}(29)-\mathrm{C}(28)-\mathrm{C}(27)$ & $119.4(4)$ \\
\hline$C(11)-C(12)-C(17)$ & $126.6(5)$ & $\mathrm{C}(29)-\mathrm{C}(28)-\mathrm{C}(33)$ & $118.2(4)$ \\
\hline $\mathrm{C}(13)-\mathrm{C}(12)-\mathrm{U}(1)$ & $74.8(3)$ & $\mathrm{C}(27)-\mathrm{C}(28)-\mathrm{C}(33)$ & $122.4(4)$ \\
\hline $\mathrm{C}(11)-\mathrm{C}(12)-\mathrm{U}(1)$ & $76.1(3)$ & $\mathrm{C}(30)-\mathrm{C}(29)-\mathrm{C}(28)$ & $121.6(5)$ \\
\hline $\mathrm{C}(17)-\mathrm{C}(12)-\mathrm{U}(1)$ & $121.2(3)$ & $\mathrm{C}(31)-\mathrm{C}(30)-\mathrm{C}(29)$ & $119.5(5)$ \\
\hline$C(12)-C(13)-C(14)$ & $108.5(5)$ & $\mathrm{C}(30)-\mathrm{C}(31)-\mathrm{C}(32)$ & $121.3(5)$ \\
\hline$C(12)-C(13)-C(18)$ & $124.8(5)$ & $C(31)-C(32)-C(27)$ & $119.6(5)$ \\
\hline$C(14)-C(13)-C(18)$ & $126.1(5)$ & $\mathrm{C}(31)-\mathrm{C}(32)-\mathrm{C}(36)$ & $119.7(4)$ \\
\hline $\mathrm{C}(12)-\mathrm{C}(13)-\mathrm{U}(1)$ & $75.2(3)$ & $\mathrm{C}(27)-\mathrm{C}(32)-\mathrm{C}(36)$ & $120.6(4)$ \\
\hline $\mathrm{C}(14)-\mathrm{C}(13)-\mathrm{U}(1)$ & $74.2(3)$ & $\mathrm{C}(28)-\mathrm{C}(33)-\mathrm{C}(34)$ & $110.4(4)$ \\
\hline $\mathrm{C}(18)-\mathrm{C}(13)-\mathrm{U}(1)$ & $124.2(3)$ & $\mathrm{C}(28)-\mathrm{C}(33)-\mathrm{C}(35)$ & $113.1(4)$ \\
\hline$C(15)-C(14)-C(13)$ & $107.0(5)$ & $\mathrm{C}(34)-\mathrm{C}(33)-\mathrm{C}(35)$ & $108.7(4)$ \\
\hline$C(15)-C(14)-C(19)$ & $127.2(5)$ & $\mathrm{C}(32)-\mathrm{C}(36)-\mathrm{C}(38)$ & $114.0(4)$ \\
\hline$C(13)-C(14)-C(19)$ & $125.6(5)$ & $\mathrm{C}(32)-\mathrm{C}(36)-\mathrm{C}(37)$ & $109.9(4)$ \\
\hline $\mathrm{C}(15)-\mathrm{C}(14)-\mathrm{U}(1)$ & $76.0(3)$ & $\mathrm{C}(38)-\mathrm{C}(36)-\mathrm{C}(37)$ & $110.8(4)$ \\
\hline $\mathrm{C}(13)-\mathrm{C}(14)-\mathrm{U}(1)$ & $75.3(3)$ & & \\
\hline
\end{tabular}

